\title{
The Global Symmetry Group of Quantum Spectral Beams and Geometric Phase Factors
}

\author{
Elias Zafiris $^{1,2}$ \\ ${ }^{1}$ Department of Mathematics, University of Athens, Panepistimioupolis, 15784 Athens, Greece \\ ${ }^{2}$ Parmenides Foundation, Kirchplatz 1, Pullach, 82049 Munich, Germany \\ Correspondence should be addressed to Elias Zafiris; ezafiris@math.uoa.gr
}

Received 10 November 2014; Accepted 9 March 2015

Academic Editor: Soheil Salahshour

Copyright ( 2015 Elias Zafiris. This is an open access article distributed under the Creative Commons Attribution License, which permits unrestricted use, distribution, and reproduction in any medium, provided the original work is properly cited.

\begin{abstract}
We propose a cohomological modelling schema of quantum state spaces and their connectivity structures in relation to the formulation of global geometric phase phenomena. In the course of this schema, we introduce the notion of Hermitian differential line sheaves or unitary rays and classify their gauge equivalence classes in terms of a global differential invariant given by the de Rham cohomology class of the curvature. Furthermore, we formulate and interpret physically the curvature recognition integrality theorem for unitary rays. Using this recognition theorem, we define the notion of a quantum spectral beam and show that it has an affine space structure with structure group given by the characters of the fundamental group.
\end{abstract}

\section{Introduction}

In the foundation of quantum mechanics, it is commonly believed that phases are not important because a state is not actually described by a vector but by a ray or a projection operator so that it can always be removed by a local phase or gauge transformation. This fact constitutes a particular manifestation of the gauge principle in quantum mechanics and it is intrinsically related to the local abelian phase invariance in the specification of the state vector of a quantum system. The locality demand in the formulation of the gauge principle is crucial because, despite the freedom of eliminating the phase locally by means of a suitable gauge transformation, global phase factors contain gauge invariant physical information and cannot be removed. Moreover, due to the Born rule probability interpretation of a state vector at a single moment of time, physical significance has been assigned only to the modulus or magnitude of a state vector whereas its phase has been ignored. Again, although it is true that the notion of phase can be always gauged away locally, this is not the case globally. Actually the typical global quantum mechanical observables are relative phases obtained by interference phenomena. These phenomena involve various splitting and recombination processes of beams whose global coherence is measured precisely by some relative phase difference. For example, we may think of the simplest case of a beam which is split into two beams propagating for a period of time and finally recombined. Their interference is always measured by a global relative phase difference. Generally, a relative phase can be thought of as the physical attribute measuring the global coherence between two beams sharing a common initial and final point in the space of some control variables parameterizing the dynamical evolution. We note that, due to the functional dependence of the dynamical evolution on the control variables, the state of a quantum system is parameterized implicitly by some temporal parameter through the control variables. Consequently, it is instructive to stress the fact that although quantum mechanics may be locally interpreted in terms of probabilities of events (so that phases do not play any role), globally it is the relative phase differences between beams that have the major physical significance. Failure to recognize this subtle point focusing on the distinctive role of the local and global levels of quantum mechanical description in relation to physical observability and classification of measurable information can cause serious interpretational problems.

The notion of a global geometric phase factor has been introduced in quantum mechanics by Berry [1] and formulated in fiber bundle theoretic terms by Simon [2]. The related notion of a global topological phase factor has been 
introduced previously by Aharonov and Bohm [3, 4]; see also [5]. The conceptual precursors of this astonishing discovery, which has been unnoticed in the foundations of quantum theory for more than 60 years, are the work of Pancharatnam in polarization optics and the Aharonov-Bohm effect in electromagnetism. In 1956 Pancharatnam [6] realized that in order to understand interference phenomena it is not required to know the absolute phase but only the relative phase difference between light beams in different states of polarization. For two light beams this relative phase is given by the phase argument of their complex-valued scalar inner product. In 1959 Aharonov and Bohm showed that there exists a measurable global phase shift in the interference pattern of two coherent wave functions of a charged particle due to the existence of an electromagnetic field constrained within an infinitely long solenoid even in case that the wave functions are localized within regions outside the solenoid, where the strength of the electromagnetic field vanishes.

The general Berry-generation mechanism of an experimentally observable global phase factor, which is of a geometric or topological origin, can be described briefly as follows. A quantum system undergoing a slowly evolving (adiabatic) cyclic evolution retains a trace of its motion after coming back to its original physical state. This trace is expressed by means of a complex phase factor in the wave function (state vector) of the system, called Berry's phase or the geometric phase. The cyclic evolution, which is better to be thought of as a periodicity property of the state vector of a quantum system, is driven by a Hamiltonian bearing an implicit time dependence through a set of control variables. For instance, we may think of external electric or magnetic fields which define the Hamiltonian parametric dependence of a charged particle. The adiabatic condition defines a constraint of parallel transport specified by the requirement that the implicit time dependence of the Hamiltonian is sufficiently slow so that the state vector stays in the eigenspace of the same instantaneous eigenvalue of the Hamiltonian. Intuitively, once the state vector is prepared in an instantaneous eigenstate of the Hamiltonian with an eigenvalue which is separated from the neighboring eigenstates by a finite energy gap, then it remains there during its transport within a finite temporal period. We may think of the space of control variables as a slowly varying environment with respect to which a state vector (eigenvector of the Hamiltonian localized at the corresponding eigenspace) displays a history dependent geometric effect. When the environment returns to its original state, the system also does, but for an additional global topological or geometric phase factor. Due to the implicit temporal dependence imposed by the time parameterization of a closed path in the environmental parameters of the control space, this global geometric phase factor is thought of as a trace of the motion encoding the global geometric or topological features of the control space. The Berry phase is a complex number of modulus one and is experimentally observable. The two most important features regarding the experimental detection of a quantum global phase are as follows: (i) it is a statistical object and (ii) it can be measured only relatively. Thus it becomes observable by comparing the historical evolution of two distinct statistical ensembles of systems through their interference pattern. The Berry phase is geometric or topological because it depends solely on the geometry or topology of the control space pathway along which the state vector is transported. It does not depend neither on the temporal metric duration of the evolution nor on the particular dynamics that is applied to the system.

It is instructive to distinguish briefly between geometric and topological global phase factors as follows. In the case of a simply connected base space of control variables, for example, a sphere, the global observable phase factor is of a geometric origin. More precisely, it appears due to the curvature of the nonintegrable connection via which the transport of state vectors takes place. In the case of a nonsimply connected space of control variables, a global observable phase factor of a topological origin is always going to appear, even if the connection is integrable. The integrability property means that the connection does not depend on the closed path traced on the control space under the proviso that these closed paths belong to the same homotopy equivalence class. Intuitively speaking, a closed path is homotopically nontrivial if it encloses a hole (e.g., an inaccessible region) so that it cannot be contracted continuously to a point in the control space. Depending on the topological connectivity properties of this space, closed paths can be classified homotopically by means of global invariants, for example, the winding number of a closed path.

The particular significance of the concepts of relative topological and geometric phase factors from the viewpoint of our theoretical scheme is that they mark a distinctive point in the history of quantum theory, where for the first time the significance of global observables as distinct entities from local observables is realized and made explicit through precise physical models, which have found concrete experimental applications. In particular, these global observables, for example, global relative phases, are obtained via an integration procedure of some differential gauge potential over a contour, represented as a closed path or loop on a base space of control variables (or localization space), which is implicitly parameterized continuously by an external temporal parameter. This is usually referred to as a cyclic evolution of a quantum state over a space of parameters, which is implicitly a continuous or smooth function of time. It is also assumed that this evolution is driven by a Hamiltonian which is expressed as a function of the localization space parameters, bearing an implicit time dependence by considering variation of the temporal parameter. Intuitively, loops in the space of control variables have the potential to probe the geometrically or topologically invariant information of the localization space, for example, by encircling a hole or an inaccessible region. This nontrivial geometric or topological information of global significance is measured in terms of global holonomy phase factors via the procedure of lifting closed paths from the localization base space to the states or observables defined over it according to some parallel transport constraint (like the adiabatic one), technically called a connection. Due to the implicit time dependence parameterizing this procedure, if we trace continuously a loop on the base space, then this loop can be lifted to the implicitly 
evolving states, represented as sections of a fiber bundle or more generally of a fibration over the base space. The particular global transformation (group element) undergone, for instance, by a state vector when it is parallel-transported along a closed curve on the localization space is called the holonomy of the connection. Thus, in this case the holonomy describes the global state vector transformation induced by cyclic changes (loops) in the controlling variables.

The first fiber bundle theoretic model of Berry's mechanism along the previous lines has been formulated by Simon [2] in terms of line bundles. Since then, almost all of the mathematical treatments of geometric phase phenomena that we are aware of have been formulated exclusively in fiber bundle terms; see, for instance, the recent collective work [7]. In this communication, we would like to propose a change of perspective from the line bundle models to the sheaves of their sections. There are two fundamental reasons justifying this move. First, the physical information is encoded functionally always in the sections of bundles and the crucial property is that the sections form sheaves, that is, implicate the manner by which local structural information can be amalgamated into global ones. Second, the usual analytic methods can be effectively formulated in terms of sheaf cohomology $[8,9]$, which may be conceived as a universal method of finding global invariants of a physical character in a homotopy-invariant way. More precisely, the sheaf cohomology groups measure the global obstructions for extending sections from the local to the global level, for example, extending local solutions of a differential equation to a global solution. The focus of this work is to demonstrate that a sheaf cohomological understanding of the origin of global geometric phase factors is physically significant and, in particular, paves the way for understanding the global symmetry group of a quantum spectral beam. For matters of space, we assume some basic familiarity of the reader with the notion of a sheaf in topology and geometry [814]. The utilization of the methods of sheaf theory and sheaf cohomology to capture the essential aspects of gauge theories, namely, electromagnetism and Yang-Mills theories has been elaborated in detail by Mallios [15, 16], inspired by the approach suggested by Selesnick [17]. We employ similar sheaf-cohomological concepts targeting the crucial gauge theoretic aspect of quantum mechanics, namely, the local phase invariance of a quantum state vector, in relation to the observation of global gauge-invariant topological and geometric phase factors accompanying the periodic evolution over a space of control variables.

\section{Cohomological Description of Quantum State Spaces}

2.1. Line Sheaves of States. From a physical viewpoint, the construction of a sheaf constitutes the natural outcome of a complete localization process [18-20]; see also [21]. Generally speaking, a localization process is being implemented in terms of an action of some category of reference or parameterizing frames on a set containing the information of some physical attribute. This set usually is endowed with some appropriate algebraic structure; for instance, we may think of a vector space of states or an algebra of observables. For example, we may consider the localization process of the set (Hilbert space basis of vectors) of the Hamiltonian eigenstates of an electron (energy eigenfunctions) with respect to some base space of control parameters. In solid state physics, the localizing base space is considered to be the momentum space [22]. More concretely, in solids the energy spectrum of the Hamiltonian operator has a band structure, meaning that it is piecewise continuous. The energy in each continuous piece depends on the momentum which varies in the Brillouin zone. Thus, the Brillouin zone of momentum variables constitutes the base localization space of the energy eigenstates of electrons. This is a continuous topological parameter space bearing the homotopy of a torus.

We consider a pair $(X, \mathbf{A})$ consisting of a paracompact (Hausdorff) topological space of control variables $X$, which is in general not a simply connected space, and a soft sheaf of commutative rings $\mathbf{A}$ localized over $X$, meaning that every section over some closed subset in $X$ can be extended to a section over $X$. We consider the above pair as the Gelfand spectrum [23] of a maximal commutative algebra of observables $\mathscr{A}$, which are comeasurable with the Hamiltonian of the quantum system. We note that if $\mathbb{C}$ is the field of complex numbers, then an $\mathbb{C}$-algebra $\mathscr{A}$ is a ring $\mathscr{A}$ together with a morphism of rings $\mathbb{C} \rightarrow \mathscr{A}$ (making $\mathscr{A}$ into a vector space over $\mathbb{C}$ ) such that the morphism $\mathscr{A} \rightarrow \mathbb{C}$ is a linear morphism of vector spaces. Notice that the same holds if we substitute the field $\mathbb{C}$ with any other field, for instance, the field of real numbers $\mathbb{R}$. We also assume that the stalk $\mathbf{A}_{x}$ of germs is a local commutative $\mathbb{C}$-algebra for any point $x \in X$. A typical example is the case, where $X$ is a smooth manifold of control variables and $\mathbf{A}$ is the $\mathbb{C}$-algebra sheaf of germs of smooth functions localized over $X$. Together with a $\mathbb{C}$-algebra sheaf $\mathbf{A}$ we also consider the abelian group sheaf of invertible elements of $\mathbf{A}$, denoted by $\widetilde{\mathbf{A}}$.

Definition 1. An A-module $\mathbf{E}$ is called a locally free $\mathbf{A}$-module of states of finite rank $m$, or simply a vector sheaf of states, if for any point $x \in X$ there exists an open set $U$ of $X$ such that

$$
\left.\mathbf{E}\right|_{U} \cong \bigoplus^{m}\left(\left.\mathbf{A}\right|_{U}\right):=\left(\left.\mathbf{A}\right|_{U}\right)^{m}
$$

where $\left(\left.\mathbf{A}\right|_{U}\right)^{m}$ denotes the $m$-terms direct sum of the sheaf of $\mathbb{C}$-observable algebras A restricted to $U$, for some $m \in \mathbb{N}$. We call $\left(\left.\mathbf{A}\right|_{U}\right)^{m}$ the local sectional frame or local gauge of states of E associated via the open covering $\mathcal{U}=\{U\}$ of $X$.

Definition 2. An A-module $\mathbf{E}$ is called a locally free $\mathbf{A}$-module of states of rank 1, or simply a line sheaf of states, if for any point $x \in X$ there exists an open set $U$ of $X$ such that

$$
\left.\left.\mathbf{E}\right|_{U} \cong \mathbf{A}\right|_{U}
$$

where $\left.\mathbf{A}\right|_{U}$ denotes the sheaf of $\mathbb{C}$-observable algebras $\mathbf{A}$ restricted to $U$. We call $\left.\mathbf{A}\right|_{U}$ the local sectional frame or local gauge of states of $\mathbf{E}$ associated via the open covering $\mathscr{U}=\{U\}$ of $X$. 
Furthermore, if for any point $x \in X$ there exists an open set $U$ of $X$ such that

$$
\left.\left.\mathbf{E}\right|_{U} \cong \mathbb{C}\right|_{U}
$$

and then we call any locally free $\mathbb{C}$-module $\mathbf{E}$ of rank 1 a complex linear local system of rank 1.

The notion of a vector sheaf of states generalizes the notion of a vector space of states when there exists a parametric dependence of the driving Hamiltonian of a quantum system from a topological space of control variables. It is also clear that the set of sections of a vector sheaf can be equipped locally with the structure of a Hilbert space. The crucial point is that locally every section of a vector sheaf can be written as a finite linear combination of a basis of sections with coefficients from the local algebra. For example, if $X$ is a smooth manifold of control variables and $\mathbf{A}$ is the $\mathbb{C}$-algebra or $\mathbb{R}$-algebra sheaf of germs of smooth functions on $X$, then every section can be locally written as a finite linear combination of a basis of sections obtained from the adiabatically parameterized spectral resolution of the Hamiltonian with coefficients being real-valued smooth functions. These functional coefficients stand for the parameterized eigenvalues. We note again that the time dependence is implicitly introduced via time-parameterized paths on $X$. It is also the case that the set of sections of every vector bundle on a topological space (not necessarily a smooth manifold) forms a vector sheaf of sections localized over this space. Thus, the notion of a vector sheaf of states provides the most general conceptual and technical framework to consider in a unifying way all cases, where there exists a parametric dependence of some observable (e.g., the energy represented by the Hamiltonian operator) and thus of the states expressed in the basis of eigenstates of the observable, by a space of control variables $X$. For example, if we consider the Schrödinger equation,

$$
\frac{\partial \psi}{\partial t}=\widehat{H}\left(\zeta_{i}(t)\right) \psi,
$$

where $\zeta_{i} \in X$, at any moment of time, and thus at each point of a time-parameterized path in $X$, we can choose an orthonormal basis diagonalizing the Hamiltonian operator:

$$
\widehat{H}\left(\zeta_{i}\right) \psi_{n}\left(\zeta_{i}\right)=E_{n}\left(\zeta_{i}\right) \psi_{n}\left(\zeta_{i}\right),
$$

where the $n$th functional coefficient is the $n$th $\zeta_{i}$-parameterized eigenvalue.

2.2. Local Gauge Freedom in terms of Cocycles. The main novelty of the sheaf theoretic perspective is that it naturally leads to an equivalent cohomological understanding of quantum state spaces, which captures the relevant gaugetheoretic aspects precisely by means of cocycles, according to the following.

Definition 3. Given a vector sheaf of states $\mathbf{E}$, there is specified a Čech 1-cocycle with respect to a covering $\mathscr{U}=\{U\}$ of $X$, called a coordinate 1 -cocycle in $Z^{1}(\mathcal{U}, \mathrm{GL}(m, \mathbf{A})$ ) (with values in the sheaf of germs of sections into the group $\mathrm{GL}(m, \mathbb{C}))$, as follows:

$$
\begin{aligned}
& \eta_{\alpha}:\left.\mathbf{E}\right|_{U_{\alpha}} \cong\left(\left.\mathbf{A}\right|_{U_{\alpha}}\right)^{m}, \\
& \eta_{\beta}:\left.\mathbf{E}\right|_{U_{\beta}} \cong\left(\left.\mathbf{A}\right|_{U_{\beta}}\right)^{m} .
\end{aligned}
$$

Thus, for every $x \in U_{\alpha}$ we have a stalk isomorphism:

$$
\eta_{\alpha}(x): \mathbf{E}_{x} \cong \mathbf{A}_{x}^{m}
$$

and similarly for every $x \in U_{\beta}$. If we consider that $x \in U_{\alpha} \cap U_{\beta}$, then we obtain the isomorphism:

$$
g_{\alpha \beta}(x)=\eta_{\alpha}(x)^{-1} \circ \eta_{\beta}(x): \mathbf{A}_{x}^{m} \cong \mathbf{A}_{x}^{m} .
$$

The $g_{\alpha \beta}(x)$ is thought of as an invertible matrix of germs at $x$. Consequently, $g_{\alpha \beta}$ is an invertible matrix section in the sheaf of germs $\mathrm{GL}(m, \mathbf{A})$ (taking values in the general linear group $\mathrm{GL}(m, \mathbb{C}))$. Moreover, $g_{\alpha \beta}$ satisfy the cocycle conditions $g_{\alpha \beta}$ 。 $g_{\beta \gamma}=g_{\alpha \gamma}$ on triple intersections whenever they are defined.

It is clear in this way that, in fiber-bundle theoretic terms, we obtain a vector bundle with typical fiber $\mathscr{C}^{m}$, structure group $\mathrm{GL}(m, \mathscr{C})$, whose sections form the vector sheaf of states we started with. In particular, for $m=1$, we obtain a line bundle $L$ with fiber $\mathscr{C}$, structure group $\operatorname{GL}(1, \mathscr{C}) \cong \widetilde{\mathscr{C}}$ (the nonzero complex numbers), whose sections form a line sheaf of states L. Clearly, by imposing a unitarity condition the structure group is reduced to $U(1)$. Thus, particularly in the case of a line sheaf of states we have the following.

Proposition 4. There exists a bijective correspondence between line sheaves of states $\mathbf{L}$ and Čech coordinate 1-cocycles $g_{\alpha \beta}$ with respect to an open covering $\mathcal{U}=\{U\}$ of $X$ :

$$
\mathbf{L} \longleftrightarrow\left(g_{\alpha \beta}\right) \in Z^{1}(\mathcal{U}, \widetilde{\mathbf{A}}),
$$

where $G L(1, \mathbf{A}) \cong \widetilde{\mathbf{A}}$ is the group sheaf of invertible elements of $\mathbf{A}$ (taking values in $\widetilde{\mathbb{C}}$ ), and $Z^{1}(\mathcal{U}, \widetilde{\mathbf{A}})$ is the set of coordinate 1-cocycles.

In physical terminology, a coordinate 1-cocycle affects a local frame (gauge) transformation of a vector sheaf of states E. In particular, in the case of a unitary line sheaf of states, a coordinate 1-cocycle affects a local abelian complex phase transformation, which reflects the local gauge freedom in the consideration of the phase of a quantum system.

From general Čech theory, it is well known that every 1cocycle can be conjugated with a 0 -cochain $\left(t_{\alpha}\right)$ in the set $C^{0}(\mathscr{U}, \widetilde{\mathbf{A}})$ to obtain another equivalent 1-cocycle:

$$
g_{\alpha \beta}^{\prime}=t_{\alpha} \cdot g_{\alpha \beta} \cdot t_{\beta}^{-1}
$$

If we consider the coboundary operator:

$$
\Delta^{0}: C^{0}(\mathcal{U}, \widetilde{\mathbf{A}}) \longrightarrow C^{1}(\mathcal{U}, \widetilde{\mathbf{A}})
$$


then the image of $\Delta^{0}$ gives the set of 1-coboundaries of the form $\Delta^{0}\left(t_{\alpha}^{-1}\right)$ in $B^{1}(\mathcal{U}, \widetilde{\mathbf{A}})$ :

$$
\Delta^{0}\left(t_{\alpha}^{-1}\right):=t_{\alpha} \cdot t_{\beta}^{-1} .
$$

Thus, we obtain that the 1-cocycle $g_{\alpha \beta}^{\prime}$ is equivalent to the 1cocycle $g_{\alpha \beta}$ in $Z^{1}(\mathcal{U}, \widetilde{\mathbf{A}})$ if and only if there exists a 0 -cochain $\left(t_{\alpha}\right)$ in the set $C^{0}(\mathscr{U}, \widetilde{\mathbf{A}})$, such that

$$
g_{\alpha \beta}^{\prime} \cdot g_{\alpha \beta}^{-1}=\Delta^{0}\left(t_{\alpha}^{-1}\right),
$$

where the multiplication above is meaningful in the abelian group of 1-cocycles $Z^{1}(\mathscr{U}, \widetilde{\mathbf{A}})$.

Due to the bijective correspondence of line sheaves with coordinate 1-cocycles with respect to an open covering $\mathscr{U}$, we deduce immediately the following proposition.

Proposition 5. The set of isomorphism classes of line sheaves of states over the same topological space of control variables $X$, denoted by $I s o(\mathbf{L})(X)$, is in bijective correspondence with the set of cohomology classes $H^{1}(X, \widetilde{\mathbf{A}})$ :

$$
\text { Iso }(\mathbf{L})(X) \cong H^{1}(X, \widetilde{\mathbf{A}}) \text {. }
$$

The above proposition interpreted in physical terms constitutes the cohomological formulation of the principle of local phase (gauge) invariance in the description of the state space of a quantum system. Furthermore, each equivalence class $[\mathbf{L}] \equiv \mathbf{L}$ in $\operatorname{Iso}(\mathbf{L})(X)$ has an inverse, defined by

$$
\mathbf{L}^{-1}:=\operatorname{Hom}_{\mathbf{A}}(\mathbf{L}, \mathbf{A}),
$$

where $\operatorname{Hom}_{\mathbf{A}}(\mathbf{L}, \mathbf{A}):=\mathbf{L}^{*}$ denotes the dual line sheaf of $\mathbf{L}$. This is actually deduced from the fact that we can define the tensor product of two equivalence classes of line sheaves over A so that

$$
L \otimes_{A} L^{*} \cong \operatorname{Hom}_{A}(L, L) \equiv \operatorname{End}_{A} L \cong A .
$$

Hence, we obtain the following proposition.

Proposition 6. The set of isomorphism classes of line sheaves of states over the same topological space $X, I s o(\mathbf{L})(X)$, has an abelian group structure with respect to the tensor product over the observable algebra sheaf $\mathbf{A}$, and analogously the set of cohomology classes $H^{1}(X, \widetilde{\mathbf{A}})$ is also an abelian group, where the tensor product of two line sheaves of states corresponds to the product of their respective coordinate 1-cocycles.

2.3. The Hermiticity Condition. For reasons implicated by the probabilistic interpretation of states in quantum mechanics via the Born rule, which utilizes the inner product structure of the state space, we need to focus on the case of isomorphism classes of Hermitian line sheaves [8, 14, 24] defined as follows.

Definition 7. (i) Given a line sheaf $\mathbf{L}$ on $X$, an $\mathbf{A}$-valued Hermitian inner product on $\mathbf{L}$ is a skew-A-bilinear sheaf morphism:

$$
\begin{aligned}
& \varrho: \mathbf{L} \oplus \mathbf{L} \longrightarrow \mathbf{A}, \\
& \varrho(\alpha s, \beta t)=\alpha \cdot \bar{\beta} \cdot \varrho(s, t)
\end{aligned}
$$

for any $s, t \in \mathbf{L}(U), \alpha, \beta \in \mathbf{A}(U), U$ open in $X$. Moreover, $\varrho(s, t)$ is skew-symmetric, namely, $\varrho(s, t)=\overline{\varrho(s, t)}$.

(ii) A line sheaf $\mathbf{L}$ on $X$, together with an $\mathbf{A}$-valued Hermitian inner product on $\mathbf{L}$, constitutes a Hermitian line sheaf.

A line sheaf is expressed in local coordinates bijectively in terms of a Čech coordinate 1 -cocycle $g_{\alpha \beta}$ in $Z^{1}(\mathcal{U}, \widetilde{\mathbf{A}})$ associated with the open covering $\mathcal{U}$. A Čech coordinate 1cocycle $g_{\alpha \beta}$ corresponding to a Hermitian line sheaf consists of local sections of $\mathbf{S U}(1, \mathbf{A})$, namely, the special unitary group sheaf of $\mathbf{A}$ of order 1 . This is simply a coordinate 1cocycle $g_{\alpha \beta}$ in $Z^{1}(\mathcal{U}, \widetilde{\mathbf{A}})$, such that the unitarity condition $\left|g_{\alpha \beta}\right|=1$ is satisfied. Clearly, in the case that a coordinate 1 -cocycle $g_{\alpha \beta}$ is constant, we have $g_{\alpha \beta}$ in $Z^{1}(\mathcal{U}, U(1))$ or equivalently $g_{\alpha \beta}$ in $Z^{1}\left(\mathcal{U}, \mathbb{S}^{1}\right)$.

2.4. The Exponential Sheaf Sequence and the Role of the Integers. In all cases of physical interest, the topological space $X$ of control variables is considered to be paracompact, while the observable algebra sheaf $\mathbf{A}$ is a soft sheaf meaning that every section over some closed subset in $X$ can be extended to a section over $X$. More importantly, the following short sequence of abelian group sheaves is exact, which models sheaf-theoretically the process of exponentiation [9].

Proposition 8. The exponential short sequence of abelian group sheaves is exact:

$$
0 \longrightarrow \mathbb{Z} \stackrel{\iota}{\longrightarrow} \mathbf{A} \stackrel{\exp }{\longrightarrow} \widetilde{\mathbf{A}} \longrightarrow 1
$$

where $\mathbb{Z}$ is the constant abelian group sheaf of integers (sheaf of locally constant sections valued in the group of integers), such that

$$
\operatorname{Ker}(\exp )=\operatorname{Im}(\iota) \cong \mathbb{Z}
$$

Clearly, due to the canonical imbedding of the constant abelian group sheaf $\mathbb{C}$ into $\mathbf{A}$ as well as of $\widetilde{\mathbb{C}}$ into $\widetilde{\mathbf{A}}$, we have the validity of the short exact sequence of constant abelian group sheaves.

Proposition 9. The exponential short sequence of abelian group sheaves is exact:

$$
\begin{aligned}
0 & \longrightarrow \mathbb{Z} \stackrel{\iota}{\longrightarrow} \mathbb{C} \stackrel{\exp }{\longrightarrow} \widetilde{\mathbb{C}} \longrightarrow 1, \\
\operatorname{Ker}(\exp ) & =\operatorname{Im}(\iota) \cong \mathbb{Z} .
\end{aligned}
$$

The above exponential short exact sequence can be specialized further to the following short exact sequence of constant abelian group sheaves:

$$
0 \longrightarrow \mathbb{Z} \stackrel{\iota}{\longrightarrow} \mathbb{R} \stackrel{\exp (2 \pi i)}{\longrightarrow} \mathbb{U}(1) \longrightarrow 1,
$$

where $\mathbb{R}$ is the constant abelian group sheaf of reals and $\mathbb{U}(1)$ is the abelian group sheaf of unit modulus complexes (phases). 
The fundamental significance of these three exponential short exact sequences of abelian group sheaves cannot be overestimated. In particular, we obtain a further refinement of the cohomological formulation of local phase invariance pertaining to the quantum state space, according to the following proposition.

Theorem 10. Each equivalence class of line sheaves of states in $I s o(L)(X)$ is in bijective correspondence with a cohomology class in the integral 2-dimensional cohomology group of $X$.

Proof. If we consider the exponential short exact sequence of abelian group sheaves, we immediately obtain a long exact sequence in sheaf cohomology, which due to paracompactness of $X$ is reduced to Čech cohomology. Thus, we have

$$
\begin{aligned}
\cdots & \longrightarrow H^{1}(X, \mathbb{Z}) \longrightarrow H^{1}(X, \mathbf{A}) \longrightarrow H^{1}(X, \widetilde{\mathbf{A}}) \\
& \longrightarrow H^{2}(X, \mathbb{Z}) \longrightarrow H^{2}(X, \mathbf{A}) \longrightarrow H^{2}(X, \widetilde{\mathbf{A}})
\end{aligned}
$$

Because of the fact that $\mathbf{A}$ is a soft sheaf, we have

$$
H^{1}(X, \mathbf{A})=H^{2}(X, \mathbf{A})=0
$$

and consequently we obtain

$$
0 \longrightarrow H^{1}(X, \widetilde{\mathbf{A}}) \stackrel{\delta_{c}}{\longrightarrow} H^{2}(X, \mathbb{Z}) \longrightarrow 0 .
$$

Thus, equivalently we obtain the following isomorphism of abelian groups (Chern isomorphism):

$$
\delta_{c}: H^{1}(X, \widetilde{\mathbf{A}}) \stackrel{\cong}{\longrightarrow} H^{2}(X, \mathbb{Z}) .
$$

Since we have shown previously that the set of isomorphism classes of line sheaves of states over $X$, namely, Iso(L) $(X)$, is in bijective correspondence with the abelian group of cohomology classes $H^{1}(X, \widetilde{\mathbf{A}})$, we deduce that

$$
\operatorname{Iso}(\mathbf{L})(X) \cong H^{2}(X, \mathbb{Z}) \text {. }
$$

Thus, each equivalence class of line sheaves of states is in bijective correspondence with a cohomology class in the integral 2-dimensional cohomology group of $X$.

\section{Curvature Differential Invariant and Spectral Beams}

3.1. Sheaves with Potentials. We have concluded that a line sheaf $\mathbf{L}$ of states on $X$ is classified up to isomorphism by a cohomology class $\delta_{c}(\mathbf{L})$ in the abelian group $H^{2}(X, \mathbb{Z})$. A natural question arising in this setting is if it is possible to express the global invariant information provided by the equivalent line sheaves' classifying integer cohomology class by means of a differential cohomological invariant. This differential invariant should be associated with a parallel transport rule imposed by a physically induced connectivity structure on a Hermitian line sheaf of states, in analogy to the original Berry-Simon formulation. It is important to emphasize that a particular connection on a line sheaf of states provides the means to express this global differential invariant locally, whereas the latter being a global invariant is independent of the particular means used to represent it locally. Notwithstanding this fact, a particular connection is directed by precise physical requirements associated with the dynamical evolution of states in relation to a space of control variables, and thus the attainment of this global invariant by using local differential means is of physical significance. We note that, conceptually speaking, the notion of a connection on a vector sheaf [9] is rooted on the sheaf-theoretic localization of the homological Kähler-de Rham differential mechanism of commutative algebras of observables. For an elaboration of this viewpoint in relation to physical applications the interested reader may find the literature instructive $[25,26]$.

Definition 11. A connection $\nabla_{\mathbf{E}}$ on a vector sheaf of states $\mathbf{E}$ is the following $\mathbb{C}$-linear sheaf morphism:

$$
\nabla_{\mathbf{E}}: \mathbf{E} \longrightarrow \Omega^{1}(\mathbf{A}) \otimes_{\mathbf{A}} \mathbf{E}
$$

referring to $\mathbb{C}$-vector space sheaves, such that the corresponding Leibniz condition is satisfied as follows:

$$
\nabla_{\mathbf{E}}(a \cdot s)=a \cdot \nabla_{\mathbf{E}}(s)+s \otimes d^{0}(a) .
$$

Proposition 12. (i) Every connection $\nabla_{\mathbf{E}}$, where $\mathbf{E}$ is a finite rank-n vector sheaf of states on $X$, can be decomposed locally as follows:

$$
\nabla_{\mathbf{E}}=d^{0}+\omega
$$

where $\omega=\omega_{\alpha \beta}$ denotes an $n \times n$ matrix of sections of local 1 -forms, called the matrix potential of $\nabla_{\mathbf{E}}$.

(ii) Under a change of local frame matrix $g=g_{\alpha \beta}$ the matrix potentials transform as follows:

$$
\omega^{\prime}=g^{-1} \omega g+g^{-1} d^{0} g .
$$

Proof. If we consider a coordinatizing basis of sections defined over an open cover $U$ of $X$, denoted by

$$
e^{U} \equiv\left\{U ;\left(e_{\alpha}\right)_{1 \leq \alpha \leq n}\right\}
$$

of the vector sheaf $\mathbf{E}$ of rank- $n$, called a local frame or a local gauge of $\mathbf{E}$, then every continuous local section $s \in \mathbf{E}(U)$, where $U \in \mathcal{U}$, can be expressed uniquely with respect to this local frame as a superposition:

$$
s=\sum_{\alpha=1}^{n} s_{\alpha} e_{\alpha}
$$

with coefficients $s_{\alpha}$ in $\mathbf{A}(U)$. The action of $\nabla_{\mathbf{E}}$ on these sections of $\mathbf{E}$ is expressed as follows:

$$
\begin{gathered}
\nabla_{\mathbf{E}}(s)=\sum_{\alpha=1}^{n}\left(s_{\alpha} \nabla_{\mathbf{E}}\left(e_{\alpha}\right)+e_{\alpha} \otimes d^{0}\left(s_{\alpha}\right)\right), \\
\nabla_{\mathbf{E}}\left(e_{\alpha}\right)=\sum_{\alpha=1}^{n} e_{\alpha} \otimes \omega_{\alpha \beta}, \quad 1 \leq \alpha, \beta \leq n,
\end{gathered}
$$


where $\omega=\omega_{\alpha \beta}$ denotes an $n \times n$ matrix of sections of local 1 -forms. Consequently we have

$$
\begin{aligned}
\nabla_{\mathbf{E}}(s) & =\sum_{\alpha=1}^{n} e_{\alpha} \otimes\left(d^{0}\left(s_{\alpha}\right)+\sum_{\beta=1}^{n} s_{\beta} \omega_{\alpha \beta}\right) \\
& \equiv\left(d^{0}+\omega\right)(s) .
\end{aligned}
$$

Thus, every connection $\nabla_{\mathbf{E}}$, where $\mathbf{E}$ is a finite rank- $n$ vector sheaf on $X$, can be decomposed locally as follows:

$$
\nabla_{\mathbf{E}}=d^{0}+\omega
$$

In this context, $\nabla_{\mathbf{E}}$ is identified as a covariant derivative operator acting on sections of the vector sheaf of states $\mathbf{E}$, and being decomposed locally as a sum consisting of a flat or integrable part identical with $d^{0}$ and a generally nonintegrable part $\omega$, called the local frame (gauge) matrix potential of the connection.

The behavior of the local potential $\omega$ of $\nabla_{\mathbf{E}}$ under local frame transformations constitutes the "transformation law of local potentials" and is obtained as follows. Let $e^{U} \equiv$ $\left\{U ; e_{\alpha=1, \ldots, n}\right\}$ and $h^{V} \equiv\left\{V ; h_{\beta=1, \ldots, n}\right\}$ be two local frames of $\mathbf{E}$ over the open sets $U$ and $V$ of $X$, such that $U \cap V \neq \emptyset$. Let us denote by $g=g_{\alpha \beta}$ the following change of local frame matrix:

$$
h_{\beta}=\sum_{\alpha=1}^{n} g_{\alpha \beta} e_{\alpha} .
$$

Under such a local frame transformation $g_{\alpha \beta}$, it is straightforward to obtain that the local potential $\omega$ of $\nabla_{\mathbf{E}}$ transforms as follows in matrix form:

$$
\omega^{\prime}=g^{-1} \omega g+g^{-1} d^{0} g
$$

We note that the above holds for any complex vector sheaf E. We may now specialize in the case of a line sheaf of states $\mathbf{L}$ equipped with a connection, denoted by the pair $(\mathbf{L}, \nabla)$. In this case of interest, due to the isomorphism

$$
\mathbf{L} \otimes_{\mathrm{A}} \mathrm{L}^{*} \cong \operatorname{Hom}_{\mathrm{A}}(\mathrm{L}, \mathrm{L}) \equiv \operatorname{End}_{\mathrm{A}} \mathrm{L} \cong \mathrm{A}
$$

we obtain that the local form of a connection over an open set is just a local 1-form or local potential (i.e., a local continuous section of $\Omega^{1}$ ).

Definition 13. A line sheaf of states L equipped with a connection $\nabla$ is called a line sheaf with potentials or equivalently a differential line sheaf and denoted by the pair $(\mathbf{L}, \nabla)$.

\subsection{Unitary Rays and Their Cohomological Characterization.} We recall that a line sheaf of states $\mathbf{L}$ on $X$, together with an $\mathbf{A}$ valued Hermitian inner product on $\mathbf{L}$, constitutes a Hermitian line sheaf.

Definition 14. A connection $\nabla$ on $\mathbf{L}$ is called Hermitian if it is compatible with $\varrho$ :

$$
d^{0} \varrho(s, t)=\varrho(\nabla s, t)+\varrho(s, \nabla t)
$$

for any $s, t \in \mathbf{L}(U), U$ open in $X$.
Definition 15. A Hermitian differential line sheaf, or equivalently a unitary ray, denoted by $(\mathbf{L}, \nabla, \varrho)$, is a Hermitian line sheaf equipped with a Hermitian connection.

First, we note that although we use the same symbol $\varrho$ in the second part of the above, it refers to an extension of the A-valued Hermitian inner product on $\mathbf{L}$, defined as follows:

$$
\begin{aligned}
& \varrho: \Omega(\mathbf{L}) \oplus \mathbf{L} \longrightarrow \Omega(\mathbf{L}), \\
& \varrho\left(s \otimes t, s^{\prime}\right):=\varrho\left(s, s^{\prime}\right) \cdot t
\end{aligned}
$$

for any $s, s^{\prime} \in \mathbf{L}(U), t \in \Omega(U)$, and $U$ open in $X$. Furthermore, $\Omega$ is considered to be a vector sheaf on $X$.

Second, if we have a Hermitian line sheaf $(\mathbf{L}, \varrho)$, which is also equipped with a connection $\nabla$, described in terms of local potentials $\omega=\left(\omega_{\alpha}\right)$, it is straightforward to see that the compatibility condition of the connection with the Hermitian inner product $\varrho$ is satisfied, such that $(\mathbf{L}, \nabla, \varrho)$ is a unitary ray, if and only if

$$
\omega+\bar{\omega}=\tilde{d}^{0}(\varrho)
$$

where, $\widetilde{d}^{0}$ denotes the logarithmic universal derivation of the observable abelian group sheaf $\widetilde{\mathbf{A}}$ to the abelian group sheaf of 1 -forms $\Omega^{1}$, that is, the universal morphism of abelian group sheaves,

$$
\widetilde{d}^{0}: \widetilde{\mathbf{A}} \longrightarrow \Omega^{1}
$$

defined by the relation

$$
\widetilde{d}^{0}(u):=u^{-1} \cdot d^{0}(u)
$$

for any continuous invertible local section $u \in \widetilde{\mathbf{A}}(U)$, with $U$ open set in $X$. Given the validity of the Poincaré Lemma, we also have

$$
\operatorname{Ker}\left(\widetilde{d}^{0}\right)=\widetilde{\mathbb{C}}
$$

Third, by considering the Hermitian line sheaf $(\mathbf{L}, \varrho)$ and a local frame of $\mathbf{L}$, we may apply locally the Gram-Schmidt orthonormalization procedure in our context, such that $\mathbf{L}$ has locally an orthonormal frame. Thus, with respect to this orthonormal frame of $\mathbf{L}$, we obtain

$$
\tilde{d}^{0}(\varrho)=0
$$

and finally we deduce that $\omega=-\bar{\omega}$.

Consequently, given that every Hermitian line sheaf $(\mathbf{L}, \varrho)$ admits a Hermitian connection, we have specified completely the notion of a unitary ray, denoted by $(\mathbf{L}, \nabla, \varrho)$, according to the above. In order to simplify further the notation, we denote a unitary ray, namely, a Hermitian differential line sheaf, by $\left(\mathbf{L}, \nabla_{\rho}\right)$. The next important task is to establish the local form of a unitary ray with respect to an open covering of $X$ and inversely determine the conditions that local components have to satisfy so that they constitute a unitary ray. 
Proposition 16. (i) The local form of a differential line sheaf is given by

$$
(\mathbf{L}, \nabla) \longleftrightarrow\left(g_{\alpha \beta}, \omega_{\alpha}\right) \in Z^{1}(\mathcal{U}, \widetilde{\mathbf{A}}) \times C^{0}\left(\mathcal{U}, \Omega^{1}\right) .
$$

(ii) An arbitrary pair $\left(g_{\alpha \beta}, \omega_{\alpha}\right) \in Z^{1}(\mathcal{U}, \widetilde{\mathbf{A}}) \times C^{0}\left(\mathcal{U}, \Omega^{1}\right)$ determines a differential line sheaf if

$$
\delta^{0}\left(\omega_{\alpha}\right)=\widetilde{d}^{0}\left(g_{\alpha \beta}\right) .
$$

Proof. A line sheaf is expressed in local coordinates bijectively in terms of a Čech coordinate 1-cocycle $g_{\alpha \beta}$ in $Z^{1}(\mathcal{U}, \widetilde{\mathbf{A}})$ associated with the covering $\mathcal{U}$. A connection $\nabla$ is expressed bijectively in terms of a 0 -cochain of 1 -forms, called the local potentials of the connection, and denoted by $\omega_{\alpha}$ with respect to the covering $\mathcal{U}$ of $X$; that is, $\omega_{\alpha} \in C^{0}\left(\mathcal{U}, \Omega^{1}\right)$. We deduce that the local form of a differential line sheaf is as follows:

$$
(\mathbf{L}, \nabla) \longleftrightarrow\left(g_{\alpha \beta}, \omega_{\alpha}\right) \in Z^{1}(\mathscr{U}, \widetilde{\mathbf{A}}) \times C^{0}\left(\mathcal{U}, \Omega^{1}\right) .
$$

Conversely, an arbitrary pair $\left(g_{\alpha \beta}, \omega_{\alpha}\right) \in Z^{1}(\mathcal{U}, \widetilde{\mathbf{A}}) \times$ $C^{0}\left(\mathcal{U}, \Omega^{1}\right)$ determines a differential line sheaf if the "transformation law of local potentials" is satisfied by this pair; that is,

$$
\begin{aligned}
& \omega_{\beta}=g_{\alpha \beta}^{-1} \omega_{\alpha} g_{\alpha \beta}+g_{\alpha \beta}^{-1} d^{0} g_{\alpha \beta}, \\
& \omega_{\beta}=\omega_{\alpha}+g_{\alpha \beta}^{-1} d^{0} g_{\alpha \beta} .
\end{aligned}
$$

Thus, given an open covering $\mathcal{U}=\left\{U_{\alpha}\right\}$, a 0 -cochain $\left(\omega_{\alpha}\right)$ valued in the sheaf $\Omega^{1}$ determines the local form of a connection $\nabla$ on the line sheaf $\mathbf{L}$, where the latter is expressed in local coordinates bijectively in terms of a Čech coordinate 1 -cocycle $\left(g_{\alpha \beta}\right)$ valued in $\widetilde{\mathbf{A}}$ with respect to $\mathcal{U}$, if and only if the corresponding local 1 -forms $\omega_{\alpha}$ of the 0 -cochain with respect to $\mathcal{U}$ are pairwise intertransformable (locally gaugeequivalent) on overlaps $U_{\alpha \beta}$ via the local frame transition functions (isomorphisms) $g_{\alpha \beta} \in \widetilde{\mathbf{A}}\left(U_{\alpha \beta}\right)$ according to the "transformation law of local potentials":

$$
\delta^{0}\left(\omega_{\alpha}\right)=\tilde{d}^{0}\left(g_{\alpha \beta}\right),
$$

where

$$
\widetilde{d}^{0}\left(g_{\alpha \beta}\right)=g_{\alpha \beta}^{-1} \cdot d^{0} g_{\alpha \beta}
$$

and $\delta^{0}$ denotes the 0th coboundary operator $\delta^{0}$ : $C^{0}\left(\mathcal{U}, \Omega^{1}\right) \rightarrow C^{1}\left(\mathcal{U}, \Omega^{1}\right)$, such that

$$
\delta^{0}\left(\omega_{\alpha}\right)=\omega_{\beta}-\omega_{\alpha}
$$

As a straightforward application of the above proposition, we obtain the following corollary.

Proposition 17. (i) The local form of a unitary ray is given by

$$
(\mathbf{L}, \nabla) \longleftrightarrow\left(g_{\alpha \beta}, \omega_{\alpha}\right) \in Z^{1}(\mathcal{U}, \widetilde{\mathbf{A}}) \times C^{0}\left(\mathcal{U}, \Omega^{1}\right),
$$

where $\left|g_{\alpha \beta}\right|=1$. (ii) An arbitrary pair $\left(g_{\alpha \beta}, \omega_{\alpha}\right) \in Z^{1}(\mathcal{U}, \widetilde{\mathbf{A}}) \times C^{0}\left(\mathcal{U}, \Omega^{1}\right)$ determines a unitary ray if

$$
\begin{gathered}
\delta^{0}\left(\omega_{\alpha}\right)=\widetilde{d}^{0}\left(g_{\alpha \beta}\right), \\
\omega+\bar{\omega}=\widetilde{d}^{0}(\varrho) .
\end{gathered}
$$

Proof. A Čech coordinate 1-cocycle $g_{\alpha \beta}$ of a unitary ray consists of local sections of $\mathbf{S U}(1, \mathbf{A})$, namely, the special unitary group sheaf of $\mathbf{A}$ of order 1 . This is simply a coordinate 1 -cocycle $g_{\alpha \beta}$ in $Z^{1}(\mathcal{U}, \widetilde{\mathbf{A}})$, such that the unitarity condition $\left|g_{\alpha \beta}\right|=1$ is satisfied. Clearly, in the case that a coordinate 1 -cocycle $g_{\alpha \beta}$ is constant, we have $g_{\alpha \beta}$ in $Z^{1}(\mathcal{U}, U(1))$ or equivalently $g_{\alpha \beta}$ in $Z^{1}\left(\mathcal{U}, \mathbb{S}^{1}\right)$. Moreover, given a Hermitian line sheaf $(\mathbf{L}, \varrho)$, which is also equipped with a connection $\nabla$, described in terms of local potentials $\omega=\left(\omega_{\alpha}\right) \epsilon$ $C^{0}\left(U, \Omega^{1}\right)$, the compatibility condition of the connection with the Hermitian inner product $\varrho$ is satisfied if and only if $\omega+\bar{\omega}=\widetilde{d}^{0}(\varrho)$.

\subsection{Gauge Equivalence of Unitary Rays. We consider two line} sheaves which are equivalent via an isomorphism $h: \mathbf{L} \stackrel{\cong}{\rightarrow} \mathbf{L}^{\prime}$. We would like to extend the notion of equivalence for two line sheaves equipped with a connective structure, namely, two differential line sheaves $(\mathbf{L}, \nabla)$ and $\left(\mathbf{L}^{\prime}, \nabla^{\prime}\right)$ and in particular two unitary rays.

Definition 18. Given an isomorphism $h: \mathbf{L} \stackrel{\cong}{\rightarrow} \mathbf{L}^{\prime}$ of line sheaves of states, we say that $\nabla$ is frame or gauge equivalent to $\nabla^{\prime}$ if they are conjugate connections under the action of $h$ :

$$
\nabla^{\prime}=h \cdot \nabla \cdot h^{-1}
$$

Thus, we may consider the set of equivalence classes on differential line sheaves $(\mathbf{L}, \nabla)$ under an isomorphism $h$ as previously denoted by Iso $(L, \nabla)$. It is now important to find the relation between Iso $(\mathbf{L}, \nabla)$ and the abelian group Iso $(\mathbf{L})$. For this purpose, we need to utilize the local form of a differential line sheaf $(\mathbf{L}, \nabla)$ and concomitantly the local form of a unitary ray $\left(\mathbf{L}, \nabla_{\rho}\right)$.

Next, we consider two line sheaves which are equivalent via an isomorphism $h: \mathbf{L} \stackrel{\cong}{\rightarrow} \mathbf{L}^{\prime}$, such that their corresponding connections are conjugate under the action of $h$ :

$$
\nabla^{\prime}=h \nabla h^{-1}
$$

Under these conditions the differential line sheaves $(\mathbf{L}, \nabla)$ and $\left(\mathbf{L}^{\prime}, \nabla^{\prime}\right)$ are called gauge or frame equivalent. Thus, we may consider the set of gauge equivalence classes $[(\mathbf{L}, \nabla)]$ of differential line sheaves as above, denoted by $\operatorname{Iso}(\mathbf{L}, \nabla)$.

Proposition 19. The set of gauge equivalence classes of unitary rays $I s o\left(\mathbf{L}, \nabla_{\rho}\right)$ is an abelian subgroup of the set of gauge equivalence classes of differential line sheaves Iso $(\mathbf{L}, \nabla)$, which in turn, is an abelian subgroup of the abelian group Iso(L). 
Proof. If we consider the local form of the tensor product of two gauge equivalent differential line sheaves, we have

$$
(\mathbf{L}, \nabla) \otimes_{\mathbf{A}}\left(\mathbf{L}^{\prime}, \nabla^{\prime}\right) \longleftrightarrow\left(g_{\alpha \beta} \cdot g_{\alpha \beta}^{\prime}, \omega_{\alpha}+\omega_{\alpha}^{\prime}\right)
$$

which satisfies the "transformation law of local potentials":

$$
\begin{aligned}
\tilde{d}^{0}\left(g_{\alpha \beta} \cdot g_{\alpha \beta}^{\prime}\right) & =\widetilde{d}^{0}\left(g_{\alpha \beta}\right)+\tilde{d}^{0}\left(g_{\alpha \beta}^{\prime}\right) \\
& =\delta^{0}\left(\omega_{\alpha}\right)+\delta^{0}\left(\omega_{\alpha}^{\prime}\right)=\delta^{0}\left(\omega_{\alpha}+\omega_{\alpha}^{\prime}\right) .
\end{aligned}
$$

Moreover, the inverse of a pair $\left(g_{\alpha \beta}, \omega_{\alpha}\right)$ is given by $\left(g_{\alpha \beta}^{-1},-\omega_{\alpha}\right)$, whereas the neutral element in the group $\operatorname{Iso}(\mathbf{L}, \nabla)$ is given by $\left(i d_{\alpha \beta}, 0\right)$, which corresponds to the trivial differential line sheaf $\left(\mathbf{A}, d^{0}\right)$. Clearly, since a unitary ray is a differential line sheaf such that $\left|g_{\alpha \beta}\right|=1$ the set of gauge equivalence classes of unitary rays $\operatorname{Iso}\left(\mathbf{L}, \nabla_{\varrho}\right)$ is an abelian subgroup of the set of gauge equivalence classes of differential line sheaves Iso $(\mathbf{L}, \nabla)$.

Next, we need to specify the local conditions under which pairs of the form $\left(g_{\alpha \beta}, \omega_{\alpha}\right)$ and $\left(g_{\alpha \beta}^{\prime}, \omega_{\alpha}^{\prime}\right)$ determine gauge equivalent differential line sheaves and concomitantly gauge equivalent unitary rays.

Proposition 20. For any two gauge equivalent differential line sheaves $(\mathbf{L}, \nabla)$ and $\left(\mathbf{L}^{\prime}, \nabla^{\prime}\right)$ in Iso $(\mathbf{L}, \nabla)$, the following holds: $(\mathbf{L}, \nabla)$ is equivalent to $\left(\mathbf{L}^{\prime}, \nabla^{\prime}\right)$, meaning that $h: \mathbf{L} \stackrel{\cong}{\rightarrow} \mathbf{L}^{\prime}$ and $\nabla^{\prime}=h \nabla h^{-1}$, if and only if there exists a 0 -cochain $\left(t_{a}\right) \in$ $C^{0}(\mathcal{U}, \widetilde{\mathbf{A}})=\prod_{\alpha} \widetilde{\mathbf{A}}\left(U_{\alpha}\right)$, such that

$$
\begin{aligned}
& g_{\alpha \beta}^{\prime} \cdot g_{\alpha \beta}^{-1}=\Delta^{0}\left(t_{a}^{-1}\right) \\
& \omega_{\alpha}^{\prime}-\omega_{\alpha}=\widetilde{d}^{0}\left(t_{a}^{-1}\right),
\end{aligned}
$$

where by definition the coordinate 1-cocycles of the line sheaves $\mathbf{L}$ and $\mathbf{L}^{\prime}$ associated with the common open covering $\mathcal{U}$ are given by $g_{\alpha \beta}=\phi_{\alpha} \circ \phi_{\beta}^{-1} \in Z^{1}(\mathcal{U}, \widetilde{\mathbf{A}})$ and $g_{\alpha \beta}^{\prime}=\psi_{\alpha} \circ \psi_{\beta}^{-1} \in$ $Z^{1}(\mathcal{U}, \widetilde{\mathbf{A}})$, according to the following diagram:

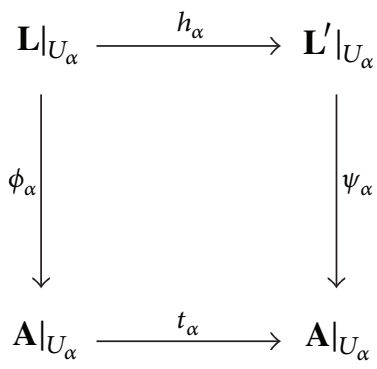

Proof. It is clear that an 1-cocycle $g_{\alpha \beta}^{\prime}$ is equivalent to another 1-cocycle $g_{\alpha \beta}$ in $Z^{1}(\mathcal{U}, \widetilde{\mathbf{A}})$ if and only if there exists a 0 cochain $\left(t_{\alpha}\right)$ in the set $C^{0}(\mathcal{U}, \widetilde{\mathbf{A}})$, such that

$$
g_{\alpha \beta}^{\prime}=t_{\alpha} \cdot g_{\alpha \beta} \cdot t_{\beta}^{-1}
$$

that is, $g_{\alpha \beta}^{\prime}$ is similar to $g_{\alpha \beta}$ under conjugation with $\left(t_{\alpha}\right)$. This is equivalently written as an action of the image of the coboundary operator, that is, of the abelian group of 1coboundaries in $B^{1}(\mathcal{U}, \widetilde{\mathbf{A}})$ on the abelian group of 1 -cocycles in $Z^{1}(\mathcal{U}, \widetilde{\mathbf{A}})$ :

$$
\begin{aligned}
\Delta^{0}: C^{0}(\mathcal{u}, \widetilde{\mathbf{A}}) & \longrightarrow C^{1}(\mathcal{U}, \widetilde{\mathbf{A}}), \\
g_{\alpha \beta}^{\prime} & =\Delta^{0}\left(t_{\alpha}^{-1}\right) \cdot g_{\alpha \beta}, \\
g_{\alpha \beta}^{\prime} \cdot g_{\alpha \beta}^{-1} & =\Delta^{0}\left(t_{\alpha}^{-1}\right) .
\end{aligned}
$$

Thus, the hypothesis of an isomorphism $h: \mathbf{L} \stackrel{\cong}{\rightarrow} \mathbf{L}^{\prime}$ is equivalent to the existence of a 0 -cochain $\left(t_{a}\right) \in C^{0}(\mathcal{U}, \widetilde{\mathbf{A}})=$ $\prod_{\alpha} \widetilde{\mathbf{A}}\left(U_{\alpha}\right)$ such that the above condition is satisfied. At a further stage, the gauge equivalence of the differential line sheaves $(\mathbf{L}, \nabla)$ and $\left(\mathbf{L}^{\prime}, \nabla^{\prime}\right)$ induced by $h \leftrightarrow\left(t_{\alpha}\right)$ implies that $\nabla^{\prime}=h \nabla h^{-1}$. Hence, by using the local expressions of the connections $\nabla^{\prime}$ and $\nabla$ by means of the local 1-forms $\omega_{\alpha}^{\prime}$ and $\omega_{\alpha}$ correspondingly, we immediately obtain

$$
\begin{aligned}
& \omega_{\alpha}^{\prime}=t_{\alpha} \omega_{\alpha} t_{\alpha}^{-1}+t_{\alpha} d^{0}\left(t_{\alpha}^{-1}\right), \\
& \omega_{\alpha}^{\prime}=\omega_{\alpha}+\widetilde{d}^{0}\left(t_{\alpha}^{-1}\right) .
\end{aligned}
$$

Thus, we have shown that

$$
\omega_{\alpha}^{\prime}-\omega_{\alpha}=\tilde{d}^{0}\left(t_{a}^{-1}\right) .
$$

3.4. De Rham Cohomology Class of the Curvature. The possibility of expressing the classifying integer cohomology class of isomorphic line sheaves of states by means of a classifying differential invariant of isomorphic differential line sheaves or unitary rays would provide a cohomological refinement of the physical gauge principle, according to which local gauge invariance implies the existence of local gauge potentials and thus of a connection on a line sheaf of states. Given that local gauge invariance is depicted by means of coordinate 1cocycles with respect to a covering $\mathcal{U}$ of $X$ and the fact that the localization of a connection is expressed in terms of local gauge potentials with respect to $\mathcal{U}$, it is natural to seek for a relation between the invariant of the former, that is, an integer cohomology class, and the differential invariant of the latter, that is, the class of the curvature of the connection. After these introductory remarks, we briefly recall the following wellknown facts.

The universal $\mathbb{C}$-derivation of the observable algebra sheaf $\mathbf{A}$ to the universal $\mathbf{A}$-module sheaf $\Omega^{1}(\mathbf{A}):=\Omega^{1}$ is the universal $\mathbb{C}$-linear sheaf morphism $d^{0}: \mathbf{A} \rightarrow \Omega^{1}$, such that the Leibniz condition $d^{0}(s \cdot t)=s \cdot d^{0}(t)+t \cdot d^{0}(s)$ is satisfied for any continuous local sections $s, t$ belonging to $\mathbf{A}(U)$, with $U$ open set in $X$. Moreover, given the validity of the Poincaré Lemma, $\operatorname{Ker}\left(d^{0}\right)=\mathbb{C}$, and the fact that $\mathbf{A}$ is a soft observable algebra sheaf, the sequence of $\mathbb{C}$-vector space sheaves is exact:

$$
\mathbf{0} \longrightarrow \mathbb{C} \longrightarrow \mathbf{A} \longrightarrow \Omega^{1}(\mathbf{A}) \longrightarrow \cdots \longrightarrow \Omega^{n}(\mathbf{A})
$$


Therefore, the sequence of $\mathbb{C}$-linear sheaf morphisms,

$$
\mathbf{A} \longrightarrow \Omega^{1}(\mathbf{A}) \longrightarrow \cdots \longrightarrow \Omega^{n}(\mathbf{A}) \longrightarrow \cdots,
$$

is a complex of $\mathbb{C}$-vector space sheaves, called the sheaftheoretic de Rham complex of $\mathbf{A}$. Thus, the sheaf-theoretic de Rham complex of the observable algebra sheaf $\mathbf{A}$ constitutes a resolution of the constant sheaf $\mathbb{C}$.

If we assume that the pair $\left(\mathbf{E}, \nabla_{\mathbf{E}}\right)$ denotes a complex vector sheaf of states equipped with a connective structure, defined by a connection $\nabla_{\mathbf{E}}$ on $\mathbf{E}$, then $\nabla_{\mathbf{E}}$ induces a sequence of $\mathbb{C}$-linear morphisms:

$$
\mathrm{E} \longrightarrow \Omega^{1}(\mathbf{A}) \otimes_{\mathbf{A}} \mathrm{E} \longrightarrow \cdots \longrightarrow \Omega^{n}(\mathbf{A}) \otimes_{\mathbf{A}} \mathbf{E} \longrightarrow \cdots
$$

or equivalently

$$
\mathrm{E} \longrightarrow \Omega^{1}(\mathrm{E}) \longrightarrow \cdots \longrightarrow \Omega^{n}(\mathbf{E}) \longrightarrow \cdots,
$$

where the morphism

$$
\nabla^{n}: \Omega^{n}(\mathbf{A}) \otimes_{\mathbf{A}} \mathbf{E} \longrightarrow \Omega^{n+1}(\mathbf{A}) \otimes_{\mathbf{A}} \mathbf{E}
$$

is given by the formula

$$
\nabla^{n}(\omega \otimes v)=d^{n}(\omega) \otimes v+(-1)^{n} \omega \wedge \nabla(v)
$$

for all $\omega \in \Omega^{n}(\mathbf{A}), v \in \mathbf{E}$. It is immediate to see that $\nabla^{0}=\nabla_{\mathbf{E}}$.

Definition 21. The composition of $\mathbb{C}$-linear morphisms $\nabla^{1} \circ \nabla^{0}$ is called the curvature of the connection $\nabla_{\mathrm{E}}$ :

$$
\nabla^{1} \circ \nabla^{0}:=\mathbf{R}_{\nabla}: \mathbf{E} \longrightarrow \Omega^{2}(\mathbf{A}) \otimes_{\mathbf{A}} \mathbf{E}=\Omega^{2}(\mathbf{E}) .
$$

As a straightforward consequence of the above definition we obtain the following.

Proposition 22. The curvature $\mathbf{R}_{\nabla}$ of a connection $\nabla_{\mathbf{E}}$ on the vector sheaf of states $\mathbf{E}$ is an $\mathbf{A}$-linear sheaf morphism, that is, a A-covariant or equivalently an A-tensor.

The A-covariant nature of the curvature $\mathbf{R}_{\nabla}$ is to be contrasted with the connection $\nabla_{\mathbf{E}}$ which is only $\mathbb{C}$-covariant. From the above, we directly derive the following corollary.

Proposition 23. The sequence of $\mathbb{C}$-linear sheaf morphisms

$$
\mathrm{E} \longrightarrow \Omega^{1}(\mathbf{A}) \otimes_{\mathrm{A}} \mathrm{E} \longrightarrow \cdots \longrightarrow \Omega^{n}(\mathbf{A}) \otimes_{\mathrm{A}} \mathrm{E} \longrightarrow \cdots
$$

is a complex of $\mathbb{C}$-vector space sheaves if and only if

$$
\mathbf{R}_{\nabla}=0 .
$$

Thus, the curvature A-linear sheaf morphism $\mathbf{R}_{\nabla}$ expresses the obstruction for the above sequence to qualify as a complex. We say that the connection $\nabla_{\mathbf{E}}$ is integrable if $\mathbf{R}_{\nabla}=0$, and we refer to the obtained complex as the sheaftheoretic de Rham complex of the integrable connection $\nabla_{\mathbf{E}}$ on the vector sheaf $\mathbf{E}$ in that case. It is also usual to call a connection $\nabla_{\mathbf{E}}$ flat if $\mathbf{R}_{\nabla}=0$. We note that the universal $\mathbb{C}$-derivation $d^{0}$ on $\mathbf{A}$ defines an integrable or flat connection.
Furthermore, we need to derive the local form of the curvature $\mathbf{R}_{\nabla}$ of a connection $\nabla_{\mathbf{E}}$, where $\mathbf{E}$ is a vector sheaf $\mathbf{E}$ on $X$. Due to its property of $\mathbf{A}$-covariance, a nonvanishing curvature represents, in this context, the A-covariant, and thus geometrically observable deviation from the unobstructed or monodromic form of variation corresponding to an integrable connection. Moreover, since the curvature $\mathbf{R}_{\nabla}$ is an $\mathbf{A}$-linear morphism of sheaves of $\mathbf{A}$-modules, that is an $\mathbf{A}$-tensor, $\mathbf{R}_{\nabla}$ may be thought of as an element of $\operatorname{End}(\mathbf{E}) \otimes_{\mathbf{A}} \Omega^{2}(\mathbf{A}):=\Omega^{2}(\operatorname{End}(\mathbf{E}))$, or equivalently, $\mathbf{R}_{\nabla} \epsilon$ $\Omega^{2}(\operatorname{End}(\mathbf{E}))$. Hence, the local form of the curvature $\mathbf{R}_{\nabla}$ of a connection $\nabla_{\mathrm{E}}$, consists of local $n \times n$ matrices having for entries local 2-forms. In particular, the local form of the curvature $\left.\mathbf{R}_{\nabla}\right|_{U}$, where $U$ is open in $X$, in terms of the local potentials $\omega$ is expressed by

$$
\left.\mathbf{R}_{\nabla}\right|_{U}=d^{1} \omega+\omega \wedge \omega
$$

as it can be easily shown by substitution of the local potentials in the composition $\nabla^{1} \circ \nabla^{0}$. Furthermore, by application of the differential operator $d^{2}$ on the above we obtain

$$
\left.d^{2} \mathbf{R}_{\nabla}\right|_{U}=\left.\mathbf{R}_{\nabla}\right|_{U} \wedge \omega-\left.\omega \wedge \mathbf{R}_{\nabla}\right|_{U} \cdot
$$

The behavior of the curvature $\mathbf{R}_{\nabla}$ of a connection $\nabla_{\mathbf{E}}$ under local frame transformations constitutes the "transformation law of potentials' strength". If we agree that $g=g_{\alpha \beta}$ denotes the change of local frame matrix we have previously considered in the discussion of the transformation law of local connection potentials, we deduce the following local transformation law:

$$
\mathbf{R}_{\nabla} \stackrel{g}{\longmapsto} \mathbf{R}_{\nabla}^{\prime}=g^{-1}\left(\mathbf{R}_{\nabla}\right) g ;
$$

that is, they transform covariantly by conjugation with respect to a local frame transformation. It is useful to collect the above findings in the form of the following proposition.

Proposition 24. (i) The local form of the curvature $\left.\mathbf{R}_{\nabla}\right|_{U}$, where $U$ is open in $X$, in terms of the local matrix potentials $\omega$ is given by

$$
\left.\mathbf{R}_{\nabla}\right|_{U}=d^{1} \omega+\omega \wedge \omega .
$$

(ii) Under a change of local frame matrix $g=g_{\alpha \beta}$ the local form of the curvature transforms by conjugation:

$$
\mathbf{R}_{\nabla} \stackrel{g}{\longmapsto} \mathbf{R}_{\nabla}^{\prime}=g^{-1}\left(\mathbf{R}_{\nabla}\right) g .
$$

We note that the above holds for any complex vector sheaf E. We may now specialize in the case of a line sheaf of states $\mathbf{L}$ equipped with a connection, denoted by the pair $(\mathbf{L}, \nabla)$. In this case of interest, due to the isomorphism,

$$
L \otimes_{A} L^{*} \cong \operatorname{Hom}_{A}(L, L) \equiv \operatorname{End}_{A} L \cong A
$$

we obtain the following simplifications: the local form of a connection over an open set is just a local 1-form or local potential (i.e., a local continuous section of $\Omega^{1}$ ), whence the local form of the curvature of the connection over an open set 
is a local 2-form. The significant result obtained by the local transformation law in this case is that the curvature is local frame invariant; that is, it does not change under any local frame (gauge) transformation:

$$
\mathbf{R}_{\nabla} \stackrel{g}{\longmapsto} \mathbf{R}_{\nabla}^{\prime}=\mathbf{R}_{\nabla}=d^{1} \omega .
$$

Thus, we obtain a global 2-form $\mathbf{R}_{\nabla}$ defined over $X$, which is also a closed 2 -form because of the fact that

$$
d^{2} \mathbf{R}_{\nabla}=0
$$

Hence, we obtain the following.

Proposition 25. For a line sheaf of states $\mathbf{L}$ equipped with a connection $\nabla$, or equivalently for a differential line sheaf $(\mathbf{L}, \nabla)$, the curvature $\mathbf{R}_{\nabla}$ of the connection is a global closed 2-form.

Clearly, the same holds true if we restrict a differential line sheaf to a unitary ray.

Proposition 26. The curvature $\mathbf{R}$ determines a global differential invariant of gauge equivalent differential line sheaves or gauge equivalent unitary rays in terms of its de Rham cohomology class $[\mathbf{R}]$.

Proof. An immediate important consequence of the characterization of gauge equivalent differential line sheaves in local terms with respect to an open covering $\mathcal{U}$ is that all of them have the same curvature. Indeed, by applying the differential operator $d^{1}$ on the above relation and using the fact that

$$
d^{1} \circ d^{0}=0
$$

from which we have that

$$
d^{1} \circ \tilde{d}^{0}=0
$$

we obtain

$$
d^{1}\left(\omega_{\alpha}^{\prime}\right)=d^{1}\left(\omega_{\alpha}\right)
$$

Hence, any two gauge equivalent differential line sheaves (and thus unitary rays) always have the same curvature, denoted by $\mathbf{R}$. We remind that $\mathbf{R}$ is a global 2 -form on $X$ since it is local frame-change invariant, which is also closed because of the fact that

$$
d^{2} \circ d^{1} \omega_{\alpha}=d^{2} \mathbf{R}=0
$$

Thus, the global 2-form $\mathbf{R}$, which belongs to $\operatorname{Ker}\left(d^{2}\right)$ : $\Omega^{2} \rightarrow \Omega^{3}$, called $\Omega_{c}^{2}$, being a $\mathbb{C}$-vector sheaf subspace of $\Omega^{2}$, determines a global differential invariant of gauge equivalent differential line sheaves. This is the case because the global 2-form $\mathbf{R}$ determines a 2-dimensional de Rham cohomology class $[\mathbf{R}]$, identified as a 2-dimensional complex Čech cohomology class in $H^{2}(X, \mathbb{C})$. In particular, if we consider a differential line sheaf the differential invariant de Rham cohomology class $[\mathbf{R}]$ is independent of the connection used to represent $\mathbf{R}$ locally. In other words, a particular connection of a differential line sheaf (or a unitary ray) provides the means to express this global differential invariant locally, whereas the latter is independent of the particular means used to represent it locally.
3.5. The Curvature Recognition Integrality Theorem. Since any two gauge equivalent differential line sheaves of states (or unitary rays) have the same curvature, we conclude that they are physically indistinguishable. This means that the abelian group $\operatorname{Iso}(\mathbf{L}, \nabla)$ is partitioned into orbits over the image of Iso $(\mathbf{L}, \nabla)$ into $\Omega_{c}^{2}$, where each orbit (fiber) is labelled by a closed 2-form $\mathbf{R}$ of $\Omega_{c}^{2}$ :

$$
\operatorname{Iso}(\mathbf{L}, \nabla)=\sum_{\mathbf{R}} \operatorname{Iso}(\mathbf{L}, \nabla)_{\mathbf{R}} \text {. }
$$

Thus, the abelian group of equivalence classes of differential line sheaves fibers over those elements of $\Omega_{c}^{2}$, namely, over those closed global 2-forms in $\Omega_{c}^{2}$ which can be identified as curvatures. In this way, a pertinent problem is the concrete identification of the image of Iso $(L, \nabla)$ into $\Omega_{c}^{2}$. Put differently, we are looking for an intrinsic characterization of those global closed 2-forms in $\Omega_{c}^{2}$, which are instantiated as curvatures of gauge equivalence classes of differential line sheaves (or unitary rays). The resolution of this problem is provided by the sheaf-theoretic formulation of the Chern-Weil integrality theorem [9]. According to this, the de Rham cohomology class $[\mathbf{R}]$ of any differential line sheaf in $H^{2}(X, \mathbb{C})$ is in the image of a cohomology class in the integral 2-dimensional cohomology group $H^{2}(X, \mathbb{Z})$ into $H^{2}(X, \mathbb{C})$.

Theorem 27. A global closed 2-form is the curvature $\mathbf{R}$ of a differential line sheaf if and only if its 2-dimensional de Rham cohomology class is integral; namely, $[\mathbf{R}] \in \operatorname{Im}\left(H^{2}(X, \mathbb{Z}) \rightarrow\right.$ $\left.H^{2}(X, \mathbb{C})\right)$.

Proof. We know that each equivalence class of line sheaves is in bijective correspondence with a cohomology class in the integral 2-dimensional cohomology group of $X$. Thus, we have to show that a 2-dimensional de Rham cohomology class is a curvature differential invariant class of gauge equivalent differential line sheaves if and only if it is an integral 2dimensional cohomology class. First, by the natural injection $\mathbb{Z} \hookrightarrow \mathbb{C}$ we obtain

$$
H^{2}(X, \mathbb{Z}) \longrightarrow H^{2}(X, \mathbb{C}),
$$

where any cohomology class belonging to the image of the above map is called an integral cohomology class. Next, we consider the following exact sequences:

$$
0 \longrightarrow \mathbb{Z} \stackrel{\iota}{\longrightarrow} \mathbf{A} \stackrel{\exp }{\longrightarrow} \widetilde{\mathbf{A}} \longrightarrow 1
$$

which is the exponential short exact sequence of abelian group sheaves, such that

$$
\operatorname{Ker}(\exp )=\operatorname{Im}(\iota) \cong \mathbb{Z}
$$

and the short exact sequence of $\mathbb{C}$-vector sheaves

$$
0 \longrightarrow \mathbb{C} \stackrel{\varepsilon}{\longrightarrow} \mathbf{A} \stackrel{d^{0}}{\longrightarrow} d^{0} \mathbf{A} \longrightarrow 0
$$

which is a fragment of the de Rham resolution of the constant sheaf $\mathbb{C}$, such that

$$
\operatorname{Ker}\left(d^{0}\right)=\operatorname{Im}(\varepsilon) \cong \mathbb{C} .
$$


Furthermore, we consider the following commutative diagram:

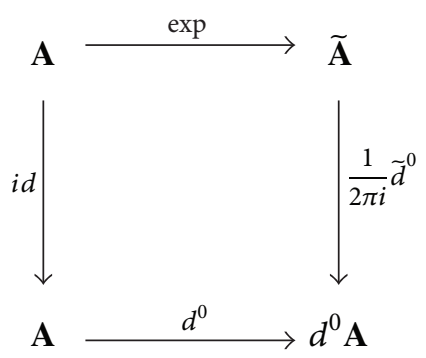

Thus we obtain the relation:

$$
2 \pi i \cdot d^{0}=\widetilde{d}^{0} \circ \exp .
$$

If we take the relevant fragments of the corresponding long exact sequences in cohomology, we obtain the following commutative diagram:

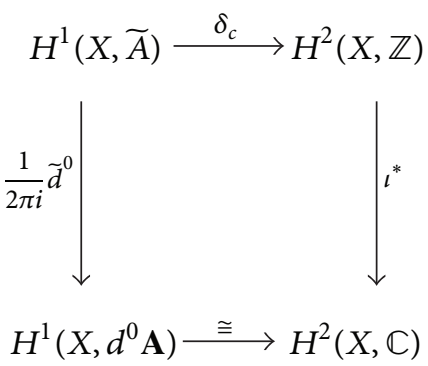

Thus, the image of a cohomology class of $H^{2}(X, \mathbb{Z})$ into an integral cohomology class of $H^{2}(X, \mathbb{C})$ corresponds to the cohomology class specified by the image of the 1-cocycle $g_{\alpha \beta}$ into $H^{1}\left(X, d^{0} \mathbf{A}\right)$, namely, by the 1-cocycle $(1 / 2 \pi i) \widetilde{d}^{0}\left(g_{\alpha \beta}\right)$.

In more detail, we first have that due to the exactness of the exponential sheaf sequence of abelian group sheaves

$$
\begin{aligned}
0 & \longrightarrow \mathbb{Z} \stackrel{\iota}{\longrightarrow} \mathbf{A} \stackrel{\exp }{\longrightarrow} \widetilde{\mathbf{A}} \longrightarrow 1 \\
g_{\alpha \beta} & =\exp \left(w_{\alpha \beta}\right),
\end{aligned}
$$

where $\left(w_{\alpha \beta}\right) \in C^{1}(\mathcal{U}, \mathbf{A})$ as well as

$$
\delta_{c}\left(w_{\alpha \beta}\right)=\left(z_{\alpha \beta \gamma}\right) \in Z^{2}(\mathcal{U}, \mathbb{Z}) .
$$

Explicitly, we may consider $w_{\alpha \beta}=\ln \left(g_{\alpha \beta}\right)$, so that

$$
\begin{aligned}
\delta_{c}\left(w_{\alpha \beta}\right) & =\left(z_{\alpha \beta \gamma}\right) \\
& :=\frac{1}{2 \pi i}\left(\ln \left(g_{\alpha \beta}\right)+\ln \left(g_{\beta \gamma}\right)-\ln \left(g_{\alpha \gamma}\right)\right) \\
& \in Z^{2}(\mathcal{U}, \mathbb{Z}) .
\end{aligned}
$$

Now, we need to locate the curvature 2-dimensional complex de Rham cohomology class differential invariant, namely, the complex 2-dimensional cohomology class $[\mathbf{R}]$ in $H^{2}(X, \mathbb{C})$. For this purpose, regarding the curvature we have that

$$
\mathbf{R}=\left(d \omega_{\alpha}\right) \in Z^{0}\left(\mathcal{U}, d \Omega^{1}\right) .
$$

By the transformation law of local potentials, it holds

$$
\delta^{0}\left(\omega_{\alpha}\right)=\tilde{d}^{0}\left(g_{\alpha \beta}\right),
$$

where

$$
\widetilde{d}^{0}\left(g_{\alpha \beta}\right)=g_{\alpha \beta}^{-1} \cdot d^{0} g_{\alpha \beta}
$$

and $g_{\alpha \beta} \in Z^{1}(\mathcal{U}, \widetilde{\mathbf{A}})$, whence $\delta^{0}$ denotes the 0 th coboundary operator $\delta^{0}: C^{0}\left(\mathcal{U}, \Omega^{1}\right) \rightarrow C^{1}\left(\mathcal{U}, \Omega^{1}\right)$, such that

$$
\delta^{0}\left(\omega_{\alpha}\right)=\omega_{\beta}-\omega_{\alpha}
$$

Thus, we obtain

$$
\delta^{0}\left(\omega_{\alpha}\right)=2 \pi i \cdot d^{0}\left(w_{\alpha \beta}\right)
$$

where $d^{0}\left(w_{\alpha \beta}\right) \in Z^{1}\left(\mathcal{U}, d^{0} \mathbf{A}\right)$. Therefore, we deduce that

$$
\frac{1}{2 \pi i}\left(\omega_{\beta}-\omega_{\alpha}\right)=d^{0}\left(\ln \left(g_{\alpha \beta}\right)\right) .
$$

If we inspect the short exact sequence of $\mathbb{C}$-vector sheaves:

$$
0 \longrightarrow \mathbb{C} \stackrel{\varepsilon}{\longrightarrow} \mathbf{A} \stackrel{d^{0}}{\longrightarrow} d^{0} \mathbf{A} \longrightarrow 0
$$

we immediately deduce that

$$
\begin{aligned}
\delta\left(\ln \left(g_{\alpha \beta}\right)\right) & =\left(\ln \left(g_{\alpha \beta}\right)+\ln \left(g_{\beta \gamma}\right)-\ln \left(g_{\alpha \gamma}\right)\right) \\
& \in Z^{2}(\mathcal{U}, \mathbb{C}) .
\end{aligned}
$$

Thus, finally we obtain

$$
\begin{gathered}
{[\mathbf{R}]=2 \pi i \cdot\left[\left(z_{\alpha \beta \gamma}\right)\right] \in H^{2}(X, \mathbb{C}),} \\
{\left[\left(g_{\alpha \beta}\right)\right]=\frac{1}{2 \pi i}[\mathbf{R}]=\left[\left(z_{\alpha \beta \gamma}\right)\right] \in H^{2}(X, \mathbb{Z}),} \\
{[\mathbf{R}] \in \operatorname{Im}\left(H^{2}(X, \mathbb{Z}) \longrightarrow H^{2}(X, \mathbb{C})\right) .}
\end{gathered}
$$

Thus, we have obtained an intrinsic characterization of the subset of those global closed 2-forms in $\Omega_{c}^{2}$, which are instantiated as curvatures of gauge equivalence classes of differential line sheaves, denoted by $\Omega_{c, \mathbb{Z}}^{2}$. Therefore, a global closed 2 -form is the curvature $\mathbf{R}$ of a differential line sheaf if and only if its 2-dimensional de Rham cohomology class is integral; namely, $[\mathbf{R}] \in \operatorname{Im}\left(H^{2}(X, \mathbb{Z}) \rightarrow H^{2}(X, \mathbb{C})\right)$.

\section{Spectral $[R]$-Beams and the Role of the Fundamental Group}

4.1. Free Action of $H^{1}\left(X, \mathbb{S}^{1}\right)$ on Spectral $[\mathbf{R}]$-Beams. From the curvature recognition integrality theorem, we have concluded that the abelian group $\operatorname{Iso}(\mathbf{L}, \nabla)$ is partitioned into orbits over $\Omega_{c, \mathbb{Z}}^{2}$, where each orbit (fiber) is labelled by an integral global closed 2-form $\mathbf{R}$ of $\Omega_{c, \mathbb{Z}}^{2}$, providing the differential invariant $[\mathbf{R}]$ of this orbit in de Rham cohomology:

$$
\operatorname{Iso}(\mathbf{L}, \nabla)=\sum_{\mathbf{R} \in \Omega_{c, \mathbb{Z}}^{2}} \operatorname{Iso}(\mathbf{L}, \nabla)_{\mathbf{R}} .
$$


If we restrict the abelian group Iso $(L, \nabla)$ to gauge equivalent Hermitian differential line sheaves (unitary rays), we obtain an abelian subgroup of the former, denoted by $\operatorname{Iso}\left(\mathbf{L}, \nabla_{\rho}\right)$. Clearly, the latter abelian group is also partitioned into orbits over $\Omega_{c, \mathbb{Z}}^{2}$, where each orbit is labelled by an integral global closed 2 -form $\mathbf{R}$ of $\Omega_{c, \mathbb{Z}}^{2}$, where $\mathbf{R}$ is the curvature of the corresponding gauge equivalence class of unitary rays.

Definition 28. We call each Hermitian differential line sheaf $\left(\mathbf{L}, \nabla_{\rho}\right)$ belonging to an orbit $\operatorname{Iso}\left(\mathbf{L}, \nabla_{\rho}\right)_{\mathbf{R}}$ a unitary $\mathbf{R}$-ray, whence the orbit itself is called a spectral $[\mathbf{R}]$-beam and is characterized by the integral differential invariant $[\mathbf{R}]$.

Each spectral [R]-beam consists of gauge equivalent unitary $\mathbf{R}$-rays, which are indistinguishable from the perspective of their common curvature integral differential invariant [R]. A natural question arising in the context of gauge equivalent unitary R-rays is how they are related to each other. In other words, although all gauge equivalent unitary $\mathbf{R}$-rays cannot be distinguished from the perspective of their curvature differential invariant, is there any other intrinsic way that we can distinguish among them? From a quantum physical perspective, if such an intrinsic and invariant way of distinguishing among gauge equivalent unitary $\mathbf{R}$-rays exists, it means that there exists a global kinematical symmetry of spectral $[\mathbf{R}]$-beams.

Proposition 29. There exists a free group action of the abelian group $H^{1}\left(X, \mathbb{S}^{1}\right)$ on the abelian group of unitary rays Iso $\left(\mathbf{L}, \nabla_{\rho}\right)$, where $\mathbb{S}^{1}$ denotes the abelian group sheaf $\mathbb{S}^{1} \equiv$ $\mathbf{U}(1) \equiv \mathbf{S} U(1, \mathbb{C})$, which is restricted to a free group action on each spectral $[\mathbf{R}]$-beam.

Proof. There exists a free group action of $\mathbb{S}^{1} \hookrightarrow \mathbb{C} \hookrightarrow \mathbf{A}$ on the group sheaf $\widetilde{\mathbf{A}}$ of invertible elements of $\mathbf{A}$ :

$$
\begin{gathered}
\mathbb{S}^{1} \times \widetilde{\mathbf{A}}(U) \longrightarrow \widetilde{\mathbf{A}}(U), \\
(\xi, f) \longmapsto \xi \cdot f
\end{gathered}
$$

with $\xi \in \mathbb{S}^{1}$ and $f \in \widetilde{\mathbf{A}}(U)$ for any open $U$ in $X$. This action is transferred naturally as a free action to the corresponding groups of coordinate 1-cocycles of the respective abelian group sheaves:

$$
\begin{aligned}
Z^{1}\left(\mathcal{U}, \mathbb{S}^{1}\right) \times Z^{1}(\mathcal{U}, \widetilde{\mathbf{A}}) & \longrightarrow Z^{1}(\mathcal{U}, \widetilde{\mathbf{A}}), \\
\left(\xi_{\alpha \beta}\right) \cdot\left(g_{\alpha \beta}\right) & =\left(\xi_{\alpha \beta} \cdot g_{\alpha \beta}\right),
\end{aligned}
$$

where $\left(\xi_{\alpha \beta}\right) \in Z^{1}\left(\mathcal{U}, \mathbb{S}^{1}\right)$ and $\left(g_{\alpha \beta}\right) \in Z^{1}(\mathcal{U}, \widetilde{\mathbf{A}})$. This free action can be also extended to the corresponding cohomology groups being still a free action:

$$
\begin{array}{r}
H^{1}\left(X, \mathbb{S}^{1}\right) \otimes H^{1}(X, \widetilde{\mathbf{A}}) \longrightarrow H^{1}(X, \widetilde{\mathbf{A}}), \\
{\left[\left(\xi_{\alpha \beta}\right)\right] \cdot\left[\left(g_{\alpha \beta}\right)\right]=\left[\left(\xi_{\alpha \beta} \cdot g_{\alpha \beta}\right)\right],}
\end{array}
$$

where $\left[\left(\xi_{\alpha \beta}\right)\right] \in H^{1}\left(X, \mathbb{S}^{1}\right)$ and $\left[\left(g_{\alpha \beta}\right)\right] \in H^{1}(X, \widetilde{\mathbf{A}}) \cong \operatorname{Iso}(\mathbf{L})$. Now, we can finally define a group action of $H^{1}\left(X, \mathbb{S}^{1}\right)$ on the abelian group Iso $(\mathbf{L}, \nabla)$ as follows. We consider $\xi \equiv\left[\left(\xi_{\alpha \beta}\right)\right] \epsilon$ $H^{1}\left(X, \mathbb{S}^{1}\right),[(\mathbf{L}, \nabla)] \in \operatorname{Iso}(\mathbf{L}, \nabla)$, and we define the sought group action as follows:

$$
\begin{aligned}
\xi \cdot[(\mathbf{L}, \nabla)] & :=[(\xi \cdot \mathbf{L}, \nabla)] \equiv\left[\left(\mathbf{L}^{\prime}, \nabla\right)\right], \\
\mathbf{L}^{\prime} & =\xi \cdot \mathbf{L} \longleftrightarrow\left(\xi_{\alpha \beta}\right) \cdot\left(g_{\alpha \beta}\right)=\left(\xi_{\alpha \beta} \cdot g_{\alpha \beta}\right) .
\end{aligned}
$$

It is immediate to show that the pair $\left(\xi_{\alpha \beta} \cdot g_{\alpha \beta}, \omega_{\alpha}\right)$ actually satisfies the "transformation law of local potentials"; that is,

$$
\delta\left(\omega_{\alpha}\right)=\tilde{d}^{0}\left(\xi_{\alpha \beta} \cdot g_{\alpha \beta}\right)
$$

Now, given that

$$
\operatorname{Ker}\left(\tilde{d}^{0}\right)=\widetilde{\mathbb{C}}
$$

as a consequence of the Poincaré Lemma, we can easily show that the above defined group action of $H^{1}\left(X, \mathbb{S}^{1}\right)$ on the abelian group Iso $(\mathbf{L}, \nabla)$ is actually free.

For this purpose, we consider the equivalent differential line sheaves $(\mathbf{L}, \nabla)$ and $\left(\mathbf{L}^{\prime}, \nabla\right)=\xi \cdot[(\mathbf{L}, \nabla)]=[(\xi \cdot \mathbf{L}, \nabla)]$ in the abelian group Iso $(\mathbf{L}, \nabla)$, where $\xi:=\left[\left(\xi_{\alpha \beta}\right)\right] \in H^{1}\left(X, \mathbb{S}^{1}\right)$, and thus $\left(\xi_{\alpha \beta}\right) \in Z^{1}\left(\mathscr{U}, \mathbb{S}^{1}\right)$, according to the above. Due to the above equivalence, we conclude that there exists a 0 -cochain $\left(t_{a}\right) \in C^{0}(\mathcal{U}, \widetilde{\mathbf{A}})=\prod_{\alpha} \widetilde{\mathbf{A}}\left(U_{\alpha}\right)$, such that

$$
\xi_{\alpha \beta}=\Delta^{0}\left(t_{\alpha}^{-1}\right)
$$

and hence, equivalently,

$$
\left[\left(\xi_{\alpha \beta}\right)\right]=1 \in H^{1}(X, \widetilde{\mathbf{A}})
$$

where, $\left(\xi_{\alpha \beta}\right) \in Z^{1}\left(\mathcal{U}, \mathbb{S}^{1}\right) \hookrightarrow\left(\xi_{\alpha \beta}\right) \in Z^{1}(\mathcal{U}, \widetilde{\mathbf{A}})$. Furthermore, by hypothesis we have

$$
\omega_{\alpha}^{\prime}=\omega_{\alpha}
$$

and thus

$$
\tilde{d}^{0}\left(t_{a}^{-1}\right)=-\tilde{d}^{0}\left(t_{a}\right)=0
$$

such that $t_{a} \in \operatorname{Ker}\left(\tilde{d}^{0}\right)=\widetilde{\mathbb{C}}$. Thus, we obtain that an automorphism $h$ of a line sheaf $\mathbf{L}$ is also an automorphism of the differential line sheaf $(\mathbf{L}, \nabla)$ if and only if $t_{a} \in \operatorname{Ker}\left(\widetilde{d}^{0}\right)=$ $\widetilde{\mathbb{C}}$. Hence, in the unitary case considered, we deduce that the 0 -cochain $\left(t_{a}\right) \in C^{0}(\mathcal{U}, \widetilde{\mathbf{A}})$ actually belongs to $C^{0}\left(\mathcal{U}, \mathbb{S}^{1}\right)$. Therefore, we conclude that

$$
\left[\left(\xi_{\alpha \beta}\right)\right]=1 \in H^{1}\left(X, \mathbb{S}^{1}\right)
$$

which proves the freeness of the above action.

We note that if we do not assume the unitarity condition, the same argument shows that the group action of $H^{1}(X, \widetilde{\mathbb{C}})$, induced by the natural injection $\widetilde{\mathbb{C}} \hookrightarrow \widetilde{\mathbf{A}}$ on the abelian group Iso $(\mathbf{L}, \nabla)$, is actually free, where in this more general case we have that $\left[\left(\xi_{\alpha \beta}\right)\right]=1 \in H^{1}(X, \widetilde{\mathbb{C}})$, where $\left(\xi_{\alpha \beta}\right) \in Z^{1}(\mathcal{U}, \widetilde{\mathbb{C}})$.

Consequently, the free group action of $H^{1}\left(X, \mathbb{S}^{1}\right)$ on Iso $(L, \nabla)$ is restricted to a free group action on its abelian 
subgroup of unitary rays Iso $\left(\mathbf{L}, \nabla_{\ell}\right)$. Since the latter abelian group is partitioned into spectral $[\mathbf{R}]$-beams over $\Omega_{c, \mathbb{Z}}^{2}$, we conclude that the above free group action is finally transferred as a free group action of $H^{1}\left(X, \mathbb{S}^{1}\right)$ on each spectral [R]beam.

Definition 30. A cohomology class in the abelian group $H^{1}\left(X, \mathbb{S}^{1}\right)$ is called a polarization phase germ of a spectral $[\mathbf{R}]$-beam.

The intuition behind the above definition is that a cohomology class in the abelian group $H^{1}\left(X, \mathbb{S}^{1}\right) \cong H^{1}(X, U(1))$ can be evaluated at a homology cycle $\gamma \in H_{1}(X)$ by means of the pairing:

$$
H_{1}(X) \times H^{1}(X, U(1)) \longrightarrow U(1)
$$

to obtain a global observable gauge-invariant phase factor in $U(1)$. Thus, gauge equivalent unitary $\mathbf{R}$-rays may be intrinsically distinguished by means of a polarization phase germ, identified as a cohomology class in $H^{1}\left(X, \mathbb{S}^{1}\right)$.

If we assume that the underlying space $X$ of control variables is a locally path-connected space, then we obtain the following.

Proposition 31. A polarization phase germ of a spectral [R]beam is realized by a representation of the fundamental group of the topological space $X$ of control variables to $\mathbb{S}^{1}$.

Proof. As an immediate consequence of the Hurewicz isomorphism we obtain

$$
\operatorname{Hom}\left(\pi_{1}(X), \widetilde{\mathbb{C}}\right) \cong H^{1}(X, \widetilde{\mathbb{C}}) .
$$

By restriction to the unitary case we obtain

$$
\operatorname{Hom}\left(\pi_{1}(X), \mathbb{S}^{1}\right) \cong H^{1}\left(X, \mathbb{S}^{1}\right) .
$$

Thus, a polarization phase germ expressed in terms of a cohomology class in $H^{1}\left(X, \mathbb{S}^{1}\right)$ is realized by a representation of the fundamental group of the topological space $X$ to $\mathbb{S}^{1}$.

4.2. Affine Space Structure of Spectral $[\mathbf{R}]$-Beams. We have shown that there exists a free group action of $H^{1}\left(X, \mathbb{S}^{1}\right) \cong$ $\operatorname{Hom}\left(\pi_{1}(X), \mathbb{S}^{1}\right)$ on each spectral $[\mathbf{R}]$-beam. A natural problem in this context is the specification of the appropriate conditions, which would qualify this free action as a transitive one as well.

For this purpose, we consider the following sequence of abelian group sheaves:

$$
1 \longrightarrow \widetilde{\mathbb{C}} \stackrel{\epsilon}{\longrightarrow} \widetilde{\mathbf{A}} \stackrel{\tilde{d}^{0}}{\longrightarrow} \Omega^{1} \stackrel{d^{1}}{\longrightarrow} d^{1} \Omega^{1} \longrightarrow 0 .
$$

As a consequence of the Poincaré Lemma we have that

$$
\operatorname{Ker}\left(\widetilde{d}^{0}\right)=\widetilde{\mathbb{C}}
$$

Moreover, we have that $d^{1} \circ \widetilde{d}^{0}=0$, and thus $\operatorname{Im}\left(\widetilde{d}^{0}\right) \subseteq$ $\operatorname{Ker}\left(d^{1}\right)$. Now, we consider those closed 1 -forms $\theta_{\alpha}$ of $\Omega^{1}$, for which the following condition is satisfied:

$$
\operatorname{Im}\left(\tilde{d}^{0}\right)=\operatorname{Ker}\left(d^{1}\right) .
$$

Definition 32. The closed 1-forms $\theta_{\alpha}$ of $\Omega^{1}$, satisfying $\operatorname{Im}\left(\tilde{d}^{0}\right)=\operatorname{Ker}\left(d^{1}\right)$, are called logarithmically exact closed 1forms.

Proposition 33. The following sequence of abelian group sheaves is an exact sequence if restricted to logarithmically exact closed 1-forms:

$$
1 \longrightarrow \widetilde{\mathbb{C}} \stackrel{\epsilon}{\longrightarrow} \widetilde{\mathbf{A}} \stackrel{\tilde{d}^{0}}{\longrightarrow} \Omega^{1} \stackrel{d^{1}}{\longrightarrow} d^{1} \Omega^{1} \longrightarrow 0 .
$$

Theorem 34. A spectral $[\mathbf{R}]$-beam is an affine space with structure group of the characters of the fundamental group with respect to logarithmically exact closed 1-forms.

Proof. By the exact sequence of abelian group sheaves of the previous proposition, we obtain a 0 -cochain $\left(\theta_{\alpha}\right)$ of logarithmically exact closed 1 -forms:

$$
\begin{aligned}
\left(\theta_{\alpha}\right) & \in C^{0}\left(\mathcal{U}, \operatorname{Ker}\left(d^{1}\right)\right)=\left(\theta_{\alpha}\right) \in C^{0}\left(\mathcal{U}, \operatorname{Im}\left(\widetilde{d}^{0}\right)\right) \\
& =\widetilde{d}^{0}\left(C^{0}(\mathcal{U}, \widetilde{\mathbf{A}})\right) .
\end{aligned}
$$

Hence, for a 0 -cochain $\left(\theta_{\alpha}\right)$ of logarithmically exact closed 1 forms $\theta_{\alpha}$, there exists a 0 -cochain $t_{\alpha}$ in $\widetilde{\mathbf{A}}$, such that

$$
\theta_{\alpha}=\widetilde{d}^{0}\left(t_{\alpha}^{-1}\right) \text {. }
$$

This 0 -cochain $\left(\theta_{\alpha}\right)$ may be considered as representing an integrable connection $\breve{\nabla}$ on a line sheaf $\mathbf{K}$, whose coordinate 1-cocycle with respect to an open covering $\mathcal{U}$ is given by

$$
\zeta_{\alpha \beta}=t_{\beta}^{-1} t_{\alpha} .
$$

Clearly, in this case the transformation laws of local potentials are satisfied as follows:

$$
\begin{aligned}
\delta^{0}\left(\theta_{\alpha}\right) & =\theta_{\beta}-\theta_{\alpha}=\widetilde{d}^{0}\left(t_{\beta}^{-1}\right)-\widetilde{d}^{0}\left(t_{\alpha}^{-1}\right)=\widetilde{d}^{0}\left(t_{\beta}^{-1} t_{\alpha}\right) \\
& =\widetilde{d}^{0}\left(\zeta_{\alpha \beta}\right) .
\end{aligned}
$$

Next, we consider a spectral [R]-beam, namely, an orbit Iso $\left(\mathbf{L}, \nabla_{\rho}\right)_{\mathbf{R}}$, consisting of gauge equivalent unitary $\mathbf{R}$-rays, which are indistinguishable from the perspective of their common differential invariant $[\mathbf{R}]$. Again, we also consider those closed 1 -forms $\theta_{\alpha}$ of $\Omega^{1}$, for which the following condition is satisfied:

$$
\operatorname{Im}\left(\widetilde{d}^{0}\right)=\operatorname{Ker}\left(d^{1}\right),
$$

namely, the logarithmically exact closed 1 -forms. We take a pair of equivalent unitary R-rays, denoted by $\left(\mathbf{L}, \nabla_{\varrho}\right),\left(\mathbf{L}^{\prime}, \nabla_{\varrho}^{\prime}\right)$ correspondingly. Then, we have that

$$
\begin{aligned}
\mathbf{R} & =\left(d \omega_{\alpha}\right)=\left(d \omega_{\alpha}^{\prime}\right), \\
d\left(\omega_{\alpha}-\omega_{\alpha}^{\prime}\right) & =0 .
\end{aligned}
$$


We conclude that $\left(\omega_{\alpha}-\omega_{\alpha}^{\prime}\right)$ is of the form $\theta_{\alpha}$ of $\Omega^{1}$, namely, it is a logarithmically exact closed 1 -form. This means that we obtain a 0 -cochain $\left(\omega_{\alpha}-\omega_{\alpha}^{\prime}\right)$ of logarithmically exact closed 1-forms:

$$
\begin{aligned}
& \left(\omega_{\alpha}-\omega_{\alpha}^{\prime}\right) \in C^{0}\left(\mathcal{U}, \operatorname{Ker}\left(d^{1}\right)\right) \\
& \quad=\left(\omega_{\alpha}-\omega_{\alpha}^{\prime}\right) \in C^{0}\left(\mathcal{U}, \operatorname{Im}\left(\widetilde{d}^{0}\right)\right)=\widetilde{d}^{0}\left(C^{0}(\mathcal{U}, \widetilde{\mathbf{A}})\right) .
\end{aligned}
$$

Hence, for a 0 -cochain $\left(\omega_{\alpha}-\omega_{\alpha}^{\prime}\right)$ of logarithmically exact closed 1-forms, there exists a 0 -cochain $t_{\alpha}$ in $\widetilde{\mathbf{A}}$, such that

$$
\begin{aligned}
\omega_{\alpha}-\omega_{\alpha}^{\prime} & =\widetilde{d}^{0}\left(t_{\alpha}^{-1}\right), \\
\omega_{\alpha} & =\omega_{\alpha}^{\prime}+\widetilde{d}^{0}\left(t_{\alpha}^{-1}\right) .
\end{aligned}
$$

Furthermore, we may consider another unitary R-ray in the same orbit, denoted by $\left(\mathbf{L}^{\prime \prime}, \nabla_{\rho}^{\prime \prime}\right)$ characterized locally as follows:

$$
\begin{aligned}
& g_{\alpha \beta}^{\prime \prime}:=\zeta_{\alpha \beta} \cdot g_{\alpha \beta}^{\prime}=t_{\alpha} \cdot g_{\alpha \beta}^{\prime} \cdot t_{\beta}^{-1}, \\
& \omega_{\alpha}^{\prime \prime}=\omega_{\alpha}=\omega_{\alpha}^{\prime}+\tilde{d}^{0}\left(t_{\alpha}^{-1}\right)
\end{aligned}
$$

such that

$$
\widetilde{d}^{0}\left(g_{\alpha \beta}^{\prime \prime}\right)=\delta^{0}\left(\omega_{\alpha}^{\prime \prime}\right) .
$$

Thus, if we consider any two unitary $\mathbf{R}$-rays $(\mathbf{L}, \nabla),\left(\mathbf{L}^{\prime}, \nabla_{\varrho}^{\prime}\right)$, we can always find another unitary $\mathbf{R}$-ray $\left(\mathbf{L}^{\prime \prime}, \nabla_{\varrho}^{\prime \prime}=\nabla_{\varrho}\right)$ equivalent to both of them, namely, belonging to the same orbit over their common differential invariant $[\mathbf{R}]$, or equivalently belonging to the same spectral $[\mathbf{R}]$-beam. Therefore, for any two unitary $\mathbf{R}$-rays $\left(\mathbf{L}, \nabla_{\varrho}\right),\left(\mathbf{L}^{\prime}, \nabla_{\varrho}^{\prime}\right)$, we can always substitute, for instance, the second one of them $\left(\mathbf{L}^{\prime}, \nabla_{\varrho}^{\prime}\right)$ with an equivalent unitary $\mathbf{R}$-ray characterized by the same 0 cochain of potentials like the first one, namely, by $\left(\mathbf{L}^{\prime \prime}, \nabla_{\rho}\right)$ whose local form is given by the above defined pair $\left(g_{\alpha \beta}^{\prime \prime}, \omega_{\alpha}\right)$. Consequently, we obtain

$$
\widetilde{d}^{0}\left(g_{\alpha \beta}^{\prime \prime}\right)=\delta^{0}\left(\omega_{\alpha}^{\prime \prime}\right)=\delta^{0}\left(\omega_{\alpha}\right)=\widetilde{d}^{0}\left(g_{\alpha \beta}\right) .
$$

We conclude that

$$
\begin{aligned}
\tilde{d}^{0}\left(g_{\alpha \beta}^{\prime \prime}\right)-\widetilde{d}^{0}\left(g_{\alpha \beta}\right) & =0, \\
\tilde{d}^{0}\left(g_{\alpha \beta}^{\prime \prime} \cdot g_{\alpha \beta}^{-1}\right) & =0 .
\end{aligned}
$$

Thus, $\left(g_{\alpha \beta}^{\prime \prime} \cdot g_{\alpha \beta}^{-1}\right) \in Z^{1}\left(\mathcal{U}, \operatorname{Ker}\left(\widetilde{d}^{0}\right)\right)$, or equivalently by the Poincaré Lemma:

$$
\left(g_{\alpha \beta}^{\prime \prime} \cdot g_{\alpha \beta}^{-1}\right) \in Z^{1}(\mathcal{U}, \widetilde{\mathbb{C}})
$$

which in the quantum unitary case considered reduces to

$$
\left(g_{\alpha \beta}^{\prime \prime} \cdot g_{\alpha \beta}^{-1}\right) \in Z^{1}\left(\mathcal{U}, \mathbb{S}^{1}\right) .
$$

The above can be equivalently formulated as follows:

$$
g_{\alpha \beta}^{\prime \prime}=\xi_{\alpha \beta} \cdot g_{\alpha \beta},
$$

where $\xi_{\alpha \beta} \in Z^{1}\left(\mathcal{U}, \mathbb{S}^{1}\right)$. By considering the corresponding cohomology classes we obtain

$$
\left[g_{\alpha \beta}^{\prime \prime}\right]=\left[\left(\xi_{\alpha \beta}\right)\right] \cdot\left[\left(g_{\alpha \beta}\right)\right]=\left[\left(\xi_{\alpha \beta} \cdot g_{\alpha \beta}\right)\right],
$$

where $\left[\left(\xi_{\alpha \beta}\right)\right] \in H^{1}\left(X, \mathbb{S}^{1}\right),\left[\left(g_{\alpha \beta}\right)\right] \in H^{1}(X, \widetilde{\mathbf{A}}) \cong \operatorname{Iso}(\mathbf{L})$. Thus, we finally deduce that

$$
\begin{aligned}
{\left[\left(\mathbf{L}^{\prime}, \nabla_{\varrho}^{\prime}\right)\right] } & =\left[\left(\mathbf{L}^{\prime \prime}, \nabla_{\varrho}\right)\right]=[(\xi \cdot \mathbf{L}, \nabla)] \\
& =[(\xi)] \cdot[(\mathbf{L}, \nabla)],
\end{aligned}
$$

where $[(\xi)]=\left[\left(\xi_{\alpha \beta}\right)\right] \in H^{1}\left(X, \mathbb{S}^{1}\right)$. Therefore, we finally arrive at the following conclusion.

The free group action of $H^{1}\left(X, \mathbb{S}^{1}\right)$ on a spectral $[\mathbf{R}]$ beam is also transitive with respect to logarithmically exact closed 1-forms, and therefore a spectral [R]-beam becomes a $H^{1}\left(X, \mathbb{S}^{1}\right) \cong \operatorname{Hom}\left(\pi_{1}(X), \mathbb{S}^{1}\right)$-affine space, or equivalently an affine space with structure group the characters of the fundamental group.

Thus, each partition block or fiber Iso $\left(\mathbf{L}, \nabla_{\rho}\right)_{\mathbf{R}}$ labelled by the curvature differential invariant $[\mathbf{R}]$, namely, each spectral $[\mathbf{R}]$-beam, is an affine space with structure group $H^{1}\left(X, \mathbb{S}^{1}\right) \cong$ $\operatorname{Hom}\left(\pi_{1}(X), \mathbb{S}^{1}\right)$. In this manner, any two unitary $\mathbf{R}$-rays differ by an element of $H^{1}\left(X, \mathbb{S}^{1}\right)$, and conversely any two unitary rays which differ by an element of $H^{1}\left(X, \mathbb{S}^{1}\right)$ are characterized by the same differential invariant $[\mathbf{R}]$ or equivalently are $\mathbf{R}$-rays of the same spectral $[\mathbf{R}]$-beam. Hence, although all gauge equivalent unitary $\mathbf{R}$-rays cannot be distinguished from the perspective of their common curvature differential invariant, there exists a free and transitive action of the group $H^{1}\left(X, \mathbb{S}^{1}\right) \cong \operatorname{Hom}\left(\pi_{1}(X), \mathbb{S}^{1}\right)$, characterized as the global kinematical symmetry group of a $[\mathbf{R}]$-beam, which completely distinguishes among them by means of characters of the fundamental group of $X$. Inversely, from any one unitary $\mathbf{R}$-ray we can obtain intrinsically its whole equivalence class by means of the free and transitive action of the abelian group $H^{1}\left(X, \mathbb{S}^{1}\right)$ on the depicted one. We conclude that whenever two unitary rays are characterized by the same differential invariant $[\mathbf{R}]$; namely, they belong to the same equivalence class (orbit) under the action of $H^{1}\left(X, \mathbb{S}^{1}\right)$ on Iso $\left(\mathbf{L}, \nabla_{\rho}\right)_{\mathbf{R}}$ (which is actually the only class due to transitivity of this action, identified as a spectral [R]beam), and then they differ by a character of the fundamental group of $X$.

\section{Conflict of Interests}

The author declares that there is no conflict of interests regarding the publication of this paper.

\section{Acknowledgment}

The paper is dedicated to the memory of Professor Anastasios Mallios, who has always insisted on the important physical 
role of differential vector sheaves in quantum theory and highlighted the essentially cohomological character of gauge theory.

\section{References}

[1] M. V. Berry, "Quantal phase factors accompanying adiabatic changes," Proceedings of the Royal Society. London. Series A. Mathematical, Physical and Engineering Sciences, vol. 392, no. 1802, pp. 45-57, 1984.

[2] B. Simon, "Holonomy, the quantum adiabatic theorem, and Berry's phase," Physical Review Letters, vol. 51, no. 24, pp. 21672170, 1983.

[3] Y. Aharonov and D. Bohm, "Significance of electromagnetic potentials in the quantum theory," vol. 115, pp. 485-491, 1959.

[4] Y. Aharonov and D. Bohm, "Further considerations on electromagnetic potentials in the quantum theory," Physical Review, vol. 123, pp. 1511-1524, 1961.

[5] F. Wilczek and A. Shapere, Geometric Phases in Physics, World Scientific, 1989.

[6] M. V. Berry, "Pancharatnam, virtuoso of the Poincaré sphere," Current Science, vol. 67, no. 4, pp. 220-223, 1994.

[7] A. Bohm, A. Mostafazadeh, H. Koizumi, Q. Niu, and J. Zwanziger, The Geometric Phase in Quantum Systems, Texts and Monographs in Physics, Springer, Berlin, Germany, 2003.

[8] A. Mallios, "Geometry of vector sheaves: an axiomatic approach to differential geometry," in Vector Sheaves, General Theory, vol. 1, Kluwer Academic Publishers, Dordrecht, The Netherlands, 1998.

[9] A. Mallios, Geometry of Vector Sheaves: An Axiomatic Approach to Differential Geometry, Vol II: Geometry Examples and Applications, vol. 439 of Mathematics and Its Applications, Kluwer Academic Publishers, Dordrecht, The Netherlands, 1998.

[10] G. E. Bredon, Topology and Geometry, Springer, New York, NY, USA, 1993.

[11] G. E. Bredon, Sheaf Theory, vol. 170 of Graduate Texts in Mathematics, Springer, Berlin, Germany, 2nd edition, 1997.

[12] E. Vassiliou, Geometry of Principal Sheaves, Kluwer Academic, Dodrecht, The Netherlands, 2004.

[13] F. Hirzebruch, Topological Methods in Algebraic Geometry, Classics in Mathematics, Springer, Berlin, Germany, 1995.

[14] S. S. Chern, Complex Manifolds without Potential Theory, Springer, New York, NY, USA, 1979.

[15] A. Mallios, Modern Differential Geometry in Gauge Theories: Vol. 1. Maxwell Fields, Birkhäuser, Boston, Mass, USA, 2006.

[16] A. Mallios, Modern Differential Geometry in Gauge Theories: Yang-Mills Fields, vol. 2, Birkhäuser, Boston, Mass, USA, 2009.

[17] S. A. Selesnick, "Line bundles and harmonic analysis on compact groups," Mathematische Zeitschrift, vol. 146, no. 1, pp. 53-67, 1976

[18] E. Zafiris, "Generalized topological covering systems on quantum events' structures," Journal of Physics A: Mathematical and General, vol. 39, no. 6, pp. 1485-1505, 2006.

[19] E. Zafiris, "Sheaf-theoretic representation of quantum measure algebras," Journal of Mathematical Physics, vol. 47, no. 9, Article ID 092103, 2006.

[20] M. Epperson and E. Zafiris, Foundations of Relational Realism: A Topological Approach to Quantum Mechanics and the Philosophy of Nature, Lexington Books, Lanham, Md, USA, 2013.
[21] A. Mallios, "On localizing topological algebras," Contemporary Mathematics, vol. 341, p. 79, 2004.

[22] R. Resta, "Manifestations of Berry's phase in molecules and condensed matter," Journal of Physics Condensed Matter, vol. 12, no. 9, pp. R107-R143, 2000.

[23] A. Mallios, Topological Algebras. Selected Topics, NorthHolland, Amsterdam, The Netherlands, 1986.

[24] P. A. Griffiths, "Hermitian differential geometry and the theory of positive and ample holomorphic vector bundles," Journal of Mathematics and Mechanics, vol. 14, pp. 117-140, 1965.

[25] A. Mallios and E. Zafiris, "The homological Kähler-de Rham differential mechanism part I: application in general theory of relativity," Advances in Mathematical Physics, vol. 2011, Article ID 191083, 14 pages, 2011.

[26] A. Mallios and E. Zafiris, “The homological Kähler-de Rham differential mechanism: II. Sheaf-theoretic localization of quantum dynamics," Advances in Mathematical Physics, vol. 2011, Article ID 189801, 13 pages, 2011. 


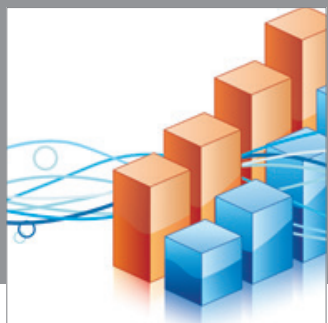

Advances in

Operations Research

mansans

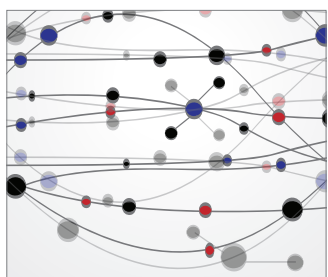

The Scientific World Journal
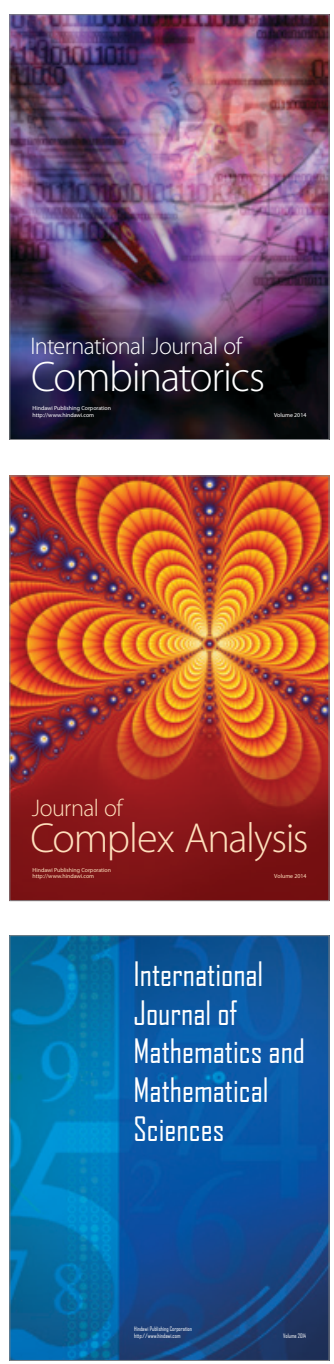
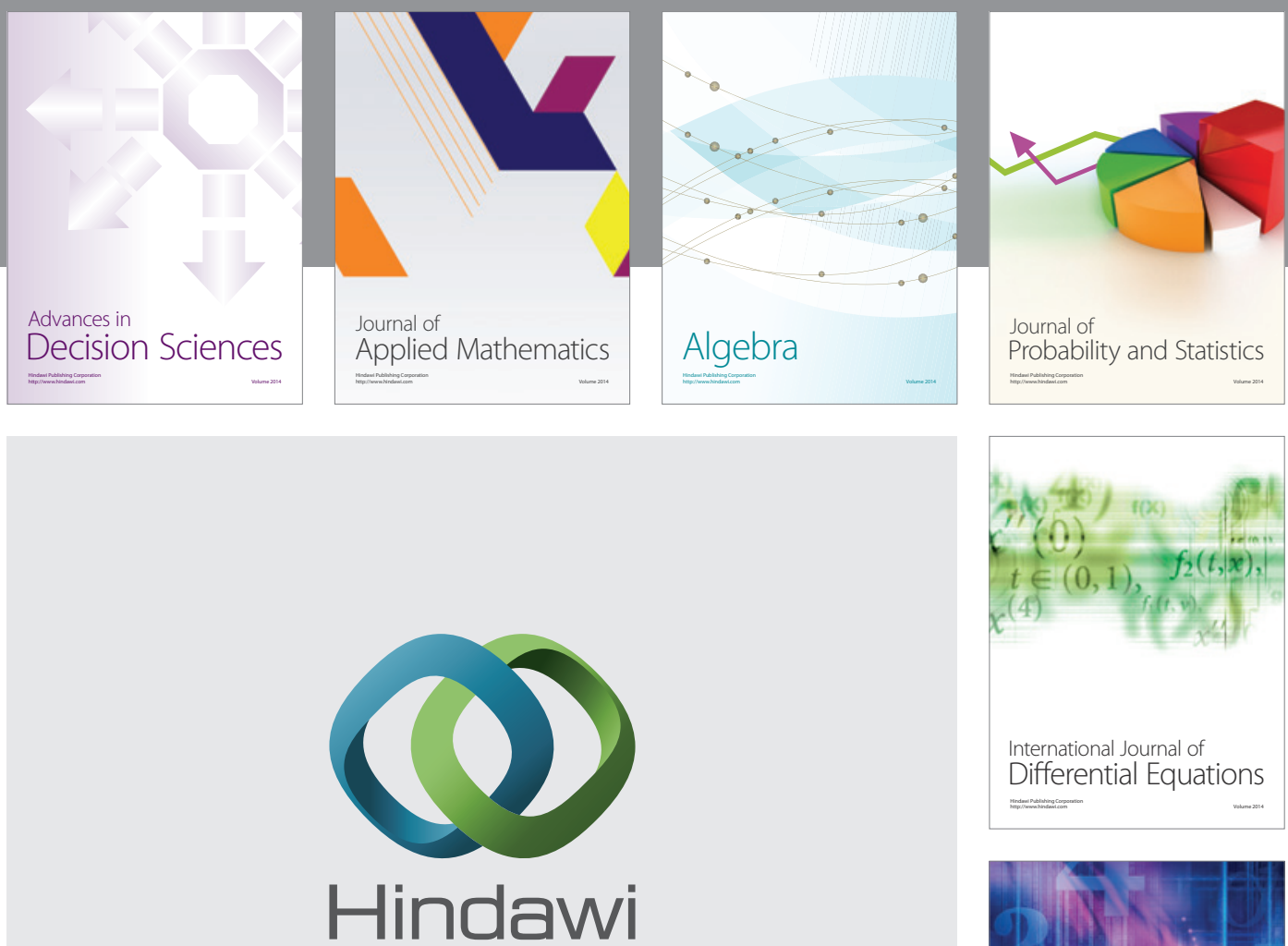

Submit your manuscripts at http://www.hindawi.com
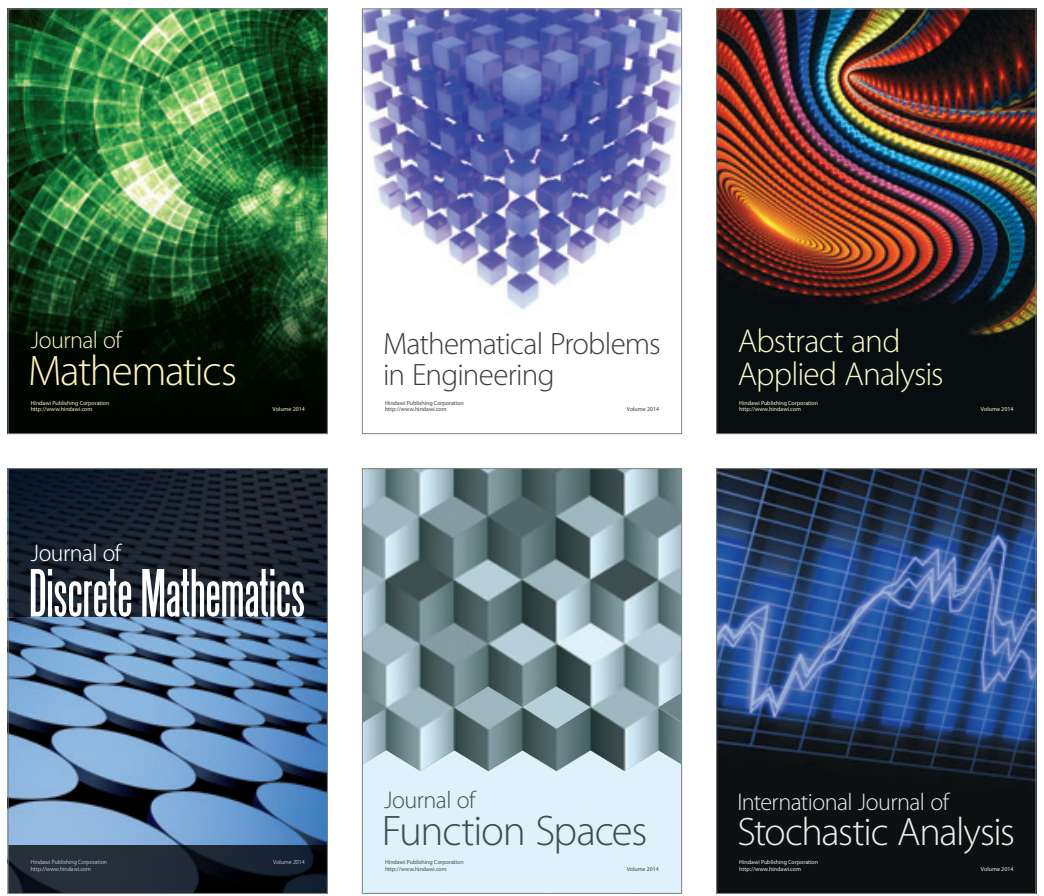

Journal of

Function Spaces

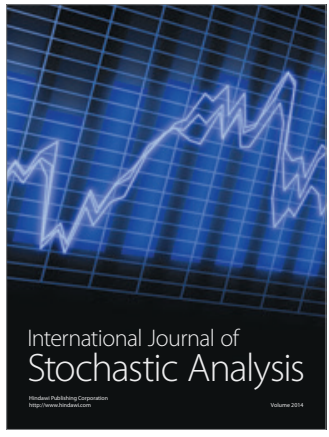

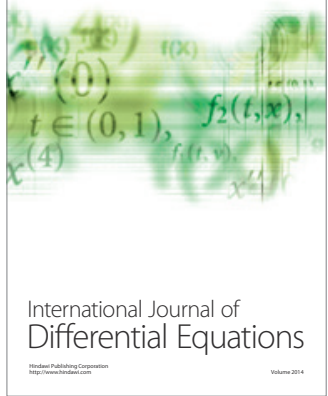
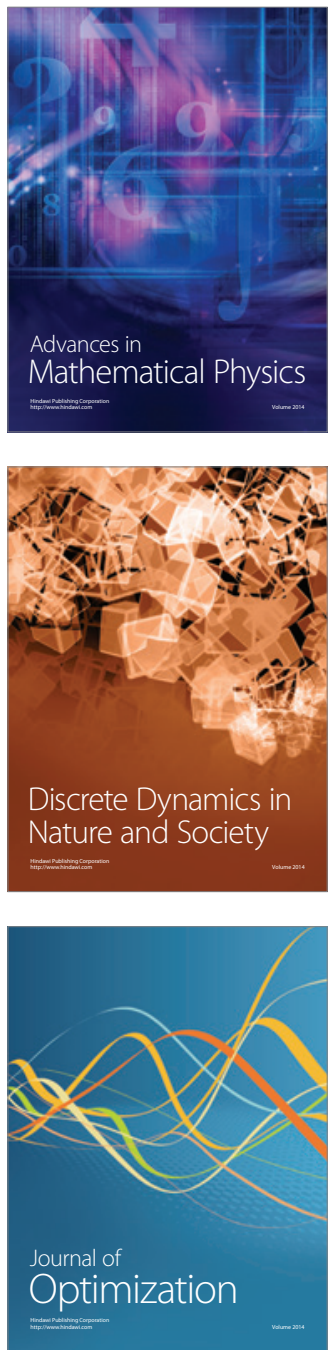